\title{
Microscopic theories of quantum lattice systems
}

\section{Document Version}

Accepted author manuscript

Link to publication record in Manchester Research Explorer

\section{Citation for published version (APA):}

Bishop, RF. (1995). Microscopic theories of quantum lattice systems. In E. Schachinger, H. Mitter, \& H. Sormann (Eds.), Recent Progress in Many-Body Theories, Vol. 4 (pp. 195-235). Plenum Publishing Corporation.

http://personalpages.manchester.ac.uk/staff/raymond.bishop/RFB_papers/[118] RPMBT_4(1995)195

\section{Published in:}

Recent Progress in Many-Body Theories, Vol. 4

\section{Citing this paper}

Please note that where the full-text provided on Manchester Research Explorer is the Author Accepted Manuscript or Proof version this may differ from the final Published version. If citing, it is advised that you check and use the publisher's definitive version.

\section{General rights}

Copyright and moral rights for the publications made accessible in the Research Explorer are retained by the authors and/or other copyright owners and it is a condition of accessing publications that users recognise and abide by the legal requirements associated with these rights.

\section{Takedown policy}

If you believe that this document breaches copyright please refer to the University of Manchester's Takedown Procedures [http://man.ac.uk/04Y6Bo] or contact uml.scholarlycommunications@manchester.ac.uk providing relevant details, so we can investigate your claim.

\section{OPEN ACCESS}




\section{Recent Progress in MANY-BODY THEORIES VOLUME 4}

Edited by

E. Schachinger

Technical University of Graz

Graz, Austria

H. Mitter

Karl-Franzen's University

Graz, Austria

and

H. Sormann

Technical University of Graz

Graz, Austria

Plenum Press - New York and London 
Proceedings of the Eighth International Conference on Recent Progress in Many-Body Theories, held August 22-26, 1994, in Liebnitz, Austria

Library of Congress Catalog Card Number 88-645051

ISBN 0-306-45103-4

(C) 1995 Plenum Press, New York

A Division of Plenum Publishing Corporation

233 Spring Street, New York, N. Y. 10013

10987654321

All rights reserved

No part of this book may be reproduced, stored in a retrieval system, or transmitted in any form or by any means, electronic, mechanical, photocopying, microfilming, recording, or otherwise, without written permission from the Publisher

Printed in the United States of America 


\title{
MICROSCOPIC THEORIES OF QUANTUM LATTICE SYSTEMS
}

\author{
Raymond F. Bishop \\ Department of Mathematics, UMIST \\ (University of Manchester Institute of Science and Technology) \\ P.O. Box 88, Manchester M60 1QD, United Kingdom
}

\section{INTRODUCTION}

Quantum-mechanical systems described by a Hamiltonian defined on an extended regular spatial lattice have become the subjects of a great deal of theoretical work in various fields of physics. Examples of considerable topical interest include: (i) spin-lattice systems such as the solid phases of ${ }^{3} \mathrm{He}$, and models of interest in magnetism, exemplified by the spin- $\frac{1}{2}$ anisotropic Heisenberg (or $X X Z$ ) model, the spin-1 Heisenbergbiquadratic model, and models with nearest and next-nearest neighbour interactions; (ii) models of strongly interacting electrons on lattices, such as the $t-J$ and Hubbard models; and (iii) lattice gauge field theories, such as the Abelian $U(1)$ and the nonAbelian $S U(2)$ models.

Until fairly recently most of the results available for such lattice systems have come from large-scale stochastic simulations using various Monte Carlo techniques; extrapolations from the exact diagonalizations of small systems; or various attempts to extend perturbation series results in the weak- and/or strong-coupling limits into the physical domain by the use of generalized Padé and similar techniques. Other approaches have included various generalizations of mean-field theory, or methods which have otherwise effectively seriously distorted the original Hamiltonian.

The study of the cuprate high-temperature superconductors provides a vivid illustration of the latter. Thus, for example, very soon after their discovery, ${ }_{1}$ it was suggested by Anderson ${ }^{2}$ that a good starting point for their description ought to be the Mott insulator, since the superconductivity occurs close to the metal-insulator transition. He further proposed that the one-band Hubbard model ${ }^{3}$ could describe the underlying physics at least qualitatively, and this remains a popular model for study. However, in his summary talk of the last but one in this series of conferences, held in 1989, Kümmel ${ }^{4}$ complained in this context that "one is forced to replace the exact Hamiltonian by a model. Finding out that this model is still not solvable, make a model of the model, then a model of the model of the model, etc., until something solvable is obtained" perhaps. It is nowadays becoming increasingly clear that what is most 
urgently needed at present is a rigorous microscopic treatment of the original Hubbard and other lattice models, despite a great deal of earlier work that attempted to circumvent this by adopting the above strategy, which has not so far proven to be notably successful.

The situation outlined above recurs frequently in the study of lattice Hamiltonian systems. It results in a common dichotomy in physics; namely, that the simplest conceptual models that one can solve are often too far removed from the underlying physical reality to be very useful, whereas even qualitative solutions to more basic but still simple models are very difficult to obtain. In the closing paragraph of his 1991 review talk on many-body problems in high-temperature superconductivity given at the last conference in this series, Lu Yu also commented ${ }^{5}$ on this same problem. $\mathrm{He}$ noted that 'historically, one may distinguish between 'conceptual' and 'quantitative' many-body theorists who do not talk to each other very much. Nowadays, this boundary becomes very fuzzy. On the one hand, the problem is so difficult that no-one now can present a convincing answer. On the other hand, the sophisticated analytic and numerical tools become more accessible to a larger group of researchers. So everybody is facing the same challenge with an equal opportunity."

In many ways these shrewd observations of Lu Yu may be regarded as the starting point for the present article. Thus, in the last few years, some of the techniques of microscopic quantum many-body theory that have proven so versatile in describing very accurately a wide range of both finite and extended systems of interest in physics and chemistry, and which are defined in continuous space, have begun to be applied to quantum lattice systems. They are now beginning to provide unified treatments of such systems, which can not only compete, for example, with other much more computationally intensive stochastic simulations, but can also provide an almost unique means to study in a systematic and unbiased manner the physically interesting quantum phase transitions.

The primary motivation of the present paper is to present an overview which stresses these new applications of such established many-body techniques as the coupled-cluster method (CCM $)^{6-15}$ and the method of correlated basis functions (CBF) ${ }^{16-23}$ To this end, the remainder of the paper is organized as follows. In order to orient the reader, we introduce in the next three Sections some typical examples of lattice Hamiltonians occurring for problems defined respectively in terms of spin operators; electron creation and destruction operators; and lattice gauge field theory operators. In the subsequent two Sections we then provide a very brief review of the main microscopic techniques that have been applied to such systems, followed by a few illustrative results that have been obtained using the CCM and CBF theory in particular. We end with some concluding remarks.

\section{TYPICAL SPIN-LATTICE MODELS}

Most spin-lattice Hamiltonians are analytically very straightforward to write down. A typical (and perhaps the earliest) example is the Heisenberg antiferromagnet. In its simplest form this is simply a system of static quantum spins on the sites of a regular $d$-dimensional lattice, interacting via nearest-neighbour interactions described by a Hamiltonian,

$$
H=J \sum_{<i, j>} \mathrm{s}_{i} \cdot \mathrm{s}_{j}
$$

with $J>0$. This system was first introduced by Heisenberg ${ }^{24}$ in the context of ferromagnetism. Dirac first showed ${ }^{25}$ how such a Hamiltonian could arise as an exchange 
interaction between atomic electrons, and since soon thereafter ${ }^{26}$ it has been of continuing interest as a model of magnetic insulators. The two-dimensional (2D) Heisenberg antiferromagnet has recently been the subject of intense renewed interest as a model ${ }^{2}$ of the insulating undoped precursors to the doped ceramic cuprates exhibiting hightemperature superconductivity. Recent reviews include those of Refs. 27 and 28.

The simplicity of form of Eq. (1) is deceptive, since it is highly nontrivial to solve in its generality. Indeed, it displays the phenomenon of exhibiting a mixture of both integrable and non-integrable types of system as various parameters are adjusted: a feature which is shared with many other spin-lattice models. Such parameters include the spin quantum number $s$, the dimensionality of the lattice and its type (e.g., square or triangular, in 2D), as well as, in the more general case, the strengths of both internal coupling parameters and any applied external fields (e.g., magnetic field). The systems often display fascinating and unexpected changes in their properties with respect to seemingly innocuous changes in these parameters. A typical example of this is provided by the Haldane conjecture ${ }^{29,30}$ for the ground state of integral-spin one-dimensional (1D) Heisenberg antiferromagnets, which has provided a second impetus for the recent resurgence of interest in spin systems.

As we shall see below, quantum spin systems typically display many interesting phase transitions. Their behaviour is often quite different from that of their classical counterparts, and rigorous theoretical results remain scarce. Even simply stated questions remain unanswered. For example, even after over 60 years of study, the question of the existence or absence of Neel order in $2 \mathrm{D}$ spin- $-\frac{1}{2}$ systems is unresolved at the level of rigorously proven results. Spin-lattice models are also of theoretical interest since they often have interesting (nonlinear field theory) continuum limits. Their approach to the quasiclassical (e.g., $s \rightarrow \infty$ ) limit is also worthy of detailed study.

Apart from their theoretical interest, spin lattice models can also be realized and studied experimentally. Apart from such obvious examples as the solid phases of ${ }^{3} \mathrm{He}$, low-dimensional systems can also be attained. For example, ${ }^{3} \mathrm{He}$ adsorbed on grafoil leads to an experimental realization of a $2 \mathrm{D}$ spin system on a triangular lattice. ${ }^{31} \mathrm{Sim}-$ ilarly, the $\mathrm{CuO}_{2}$ planes in the undoped ceramic cuprates exhibiting high-temperature superconductivity are believed to be well modelled by the spin- $\frac{1}{2} 2 \mathrm{D}$ Heisenberg model on a square lattice. The physical hypothesis is that this model may provide a description of the interactions between the electrons in the singly-occupied $d_{x^{2}-y^{2}}$ orbitals on the copper atoms.

Nowadays, there are also many materials which are known to behave to a greater or lesser degree as quasi-1D spin systems exhibiting either ferromagnetic or antiferromagnetic behaviour. For example, neutron scattering experiments on CPC (dichlorobispyridine copper), $\mathrm{CuCl}_{2} \cdot 2 \mathrm{~N}\left(\mathrm{C}_{5} \mathrm{D}_{5}\right)$, have been interpreted as providing strong evidence that this material provides a rather accurate realization of a spin- $\frac{1}{2} 1 \mathrm{D}$ (isotropic) Heisenberg antiferromagnet. Similarly, $\mathrm{CsCoCl}_{3}$ (and $\mathrm{CsCoBr}_{3}$ ) on the one hand and $\mathrm{Cs}_{2} \mathrm{CoCl}_{4}$ on the other are believed to be good physical realizations of $1 \mathrm{D}$ spin$\frac{1}{2}$ quantum antiferromagnets of anisotropic Heisenberg (or $X X Z$ ) type, showing high anisotropy respectively into the Ising-like and planar (or $X Y$ ) regimes. In the same vein, $\mathrm{CoCl}_{2} \cdot 2 \mathrm{D}_{2} \mathrm{O}$ is believed to model the comparable $1 \mathrm{D}$ spin- $\frac{1}{2}$ Ising-like quantum ferromagnet.

Other materials model $1 \mathrm{D}$ systems with higher spin values. For example, $\mathrm{CsNiCl}_{3}$ and NENP (nickel(2) ethylenediamine nitrito perchlorate), $\mathrm{Ni}\left(\mathrm{C}_{2} \mathrm{H}_{8} \mathrm{~N}_{2}\right)_{2} \mathrm{NO}_{2}\left(\mathrm{ClO}_{4}\right)$, are both quasi-1D spin-1 antiferromagnets; and $C s N i F_{3}$ is a quasi-1D spin-1 ferromagnet. All three materials are believed to be well represented by the spin- 1 Heisenberg model (with additional easy-plane crystal field anisotropy). Finally, TMMC (tetram- 
ethylammonium manganese trichloride), $\left(\mathrm{CH}_{3}\right)_{4} \mathrm{NMnCl}_{3}$, is a quasi-1D spin- $\frac{\mathbf{5}}{2}$ (and hence quasiclassical, due to the high spin value) antiferromagnet of nearly Heisenberg type, but with a small anisotropy into the planar ( $X Y$-model) regime. Many other examples also exist.

The most general spin-lattice problems comprises $N$ quantum-mechanical spins $\mathbf{s}_{k} \equiv$ $\left\{s_{k}^{a} ; a=x, y, z\right\}$ on the sites $\{k\}$ of a given regular lattice in $d$ dimensions Periodic boundary conditions are usually assumed for the lattice, and in any case we shall always be interested here in the infinite lattice limit, $N \rightarrow \infty$. The spins obey the usual $S U(2)$ angular momentum algebra,

$$
\left[s_{k}^{a}, s_{\ell}^{b}\right]=i \delta_{k \ell} s_{k}^{c},
$$

where $(a, b, c)$ is any cyclic permutation of the indices $(x, y, z)$. The spin quantum number $s$ may, in general, take any of the usual integral or half-integral values,

$$
\mathbf{s}_{k}^{2}=s(s+1) ; s=\frac{1}{2}, 1, \frac{3}{2}, \cdots .
$$

The simplest lattices are the so-called bipartite lattices. These are lattices which can be divided into two equivalent sublattices, so that all $z$ nearest neighbours of a given site on either sublattice lie on the other sublattice. Such bipartite lattices lend themselves, at least naively, to a classical picture of antiferromagnetism in which all of the spins on one sublattice have $s^{z}=+s$ and all of those on the other have $s^{z}=-s$. This is simply the classical Neel state. By contrast, lattices which do not permit this division are called frustrated. Examples in 2D include the triangular lattice and the Kagomé lattice. The latter is a lattice built of triangular plaquettes with coordination number 4 , or, equivalently, a triangular lattice from which $\frac{1}{4}$ of the lattice points, corresponding to those on a triangular lattice with twice the original spacing, have been removed.

In the remainder of this Section a number of interesting extensions of the original Heisenberg model of Eq. (1) are outlined. The simplest of these is probably the $X X Z$ or anisotropic Heisenberg model, with Hamiltonian given by

$$
H=\frac{1}{2} \sum_{\ell} \sum_{\rho}\left(s_{\ell}^{x} s_{\ell+\rho}^{x}+s_{\ell}^{y} s_{\ell+\rho}^{y}+\Delta s_{\ell}^{z} s_{\ell+\rho}^{z}\right),
$$

where the sum on the (vector) index $\ell$ runs over all lattice sites; the sum on the (vector) index $\rho$ runs over all $z$ nearest neighbour sites; $\Delta$ is the anisotropy parameter; and we have set the antiferromagnetic coupling constant $J$ to unity. This Hamiltonian contains as three special cases the Ising model $(\Delta \rightarrow \infty)$, the isotropic Heisenberg model $(\Delta=1)$, and the planar (or $X Y)$ model $(\Delta=0)$. From the commutation relations of Eq. (2), it is easy to show that

$$
\left[H, s_{T}^{z}\right]=0,
$$

for the Hamiltonian of Eq. (4) where $\mathbf{s}_{T}$ is the total spin operator,

$$
\mathbf{s}_{T}=\sum_{k=1}^{N} \mathbf{s}_{k} .
$$

The energy eigenstates of Eq. (4) may thus be chosen to be simultaneous eigenstates of the $z$-component of total spin, $s_{T}^{z}$, which is hence a good quantum number. In particular, the antiferromagnetic ground state has $s_{T}^{z}=0$.

The study of the $X X Z$ model has a long and interesting history. As early as 1931 , Bethe $^{32}$ found the exact solutions for the eigenstates of the $1 D$ spin- $\frac{1}{2}$ isotropic $(\Delta=1)$ antiferromagnetic Heisenberg model. Seven years later Hulthén ${ }^{33}$ calculated the exact 
value of the ground-state energy based on the Bethe ansatz. Since then the Bethe ansatz has been generalized to the $X X Z$ model $^{34}$ as well as to other $1 \mathrm{D}$ quantum systems. ${ }^{35}$ The excitations and other physical properties of the corresponding models have also been investigated by the Bethe ansatz and other means. ${ }^{36}$ In particular, for the $X X Z$ model, the staggered magnetization was obtained by Baxter, ${ }^{37}$ and the asymptotics of the correlation functions were obtained ${ }^{38}$ in 1986 by the algebraization of the Bethe ansatz, namely the quantum inverse scattering method. ${ }^{39}$ This latter approach has confirmed the results of earlier work by Luther and Peschel..$^{40}$

The main exact results for the spin- $\frac{1}{2} 1 \mathrm{D} X X Z$ model can be summarized as follows. In the ferromagnetic $\left(s_{T}^{z}= \pm \frac{1}{2} N\right)$ regime, $\Delta \leq-1$, the exact ground-state energy is trivially given by

$$
E_{0} / N=\frac{1}{4} \Delta ; \quad \Delta \leq-1,
$$

just as in the classical case. Precisely at $\Delta=-1$ there is a first-order phase transition to a phase with $s_{T}^{z}=0$. Such phases we henceforth refer to loosely as antiferromagnetic phases. This phase exhibits no long-range order (of the Néel type). Nevertheless, it is a critical phase in the usual sense that the spin-spin correlation function shows algebraic decaying behaviour, details of which are given below. This critical phase persists over the entire range $|\Delta| \leq 1$ of the anisotropy parameter. Since it includes the $\Delta=0$ case we also refer to it as the planar-like (or $X Y$-model-like) phase. Its ground-state energy is given by the exact expression,

$$
\begin{gathered}
\frac{E_{0}}{N}=\frac{1}{4} \cos \theta-\frac{1}{2} \sin ^{2} \theta \int_{-\infty}^{\infty} \frac{d w}{\cosh (\pi w)[\cosh (2 \theta w)-\cos \theta]} ; \quad|\Delta| \leq 1, \\
0 \leq \theta \equiv \cos ^{-1} \Delta \leq \pi .
\end{gathered}
$$

From Eq. (8) one may evaluate the energy explicitly at various special points. Thus, at $\Delta=-1, E_{0} / N=-\frac{1}{4}$; at $\Delta=0$ (i.e., the $X Y$ model), $E_{0} / N=-1 / \pi$; and at the isotropic point $\Delta=1$ (i.e., the Heisenberg model), $E_{0} / N=\frac{1}{4}-\ln 2$.

Whereas the critical phase which exists for $|\Delta|<1$ has a $\left(s_{T}^{z}=0\right)$ nondegenerate ground state, at $\Delta=1$ the system undergoes another phase change to a new Ising-like antiferromagnetic phase which persists over the entire range $\Delta>1$. This phase has a doubly degenerate ground state, but still with $s_{T}^{z}=0$. The ground-state energy and all its finite-order derivatives change continuously at $\Delta=1$. We have the exact result,

$$
\frac{E_{0}}{N}=\frac{1}{4} \cosh \gamma-\frac{1}{2} \sinh \gamma\left[1+4 \sum_{m=1}^{\infty}\left(1+\mathrm{e}^{2 m \gamma}\right)^{-1}\right] ; \quad 1 \leq \Delta \equiv \cosh \gamma,
$$

Direct evaluation from Eq. (9) shows that at $\Delta=1$ the energy has the same value as given by Eq. (8), whereas in the Ising limit $(\Delta \rightarrow \infty)$ one has

$$
\frac{E_{0}}{N} \underset{\Delta \rightarrow \infty}{\longrightarrow}-\frac{1}{4}\left(\Delta+\frac{1}{\Delta}\right)
$$

The principal difference between the two antiferromagnetic phases of the spin- $\frac{1}{2}$ $X X Z$-model chain in $1 \mathrm{D}$ is in their long-range order properties. These may conveniently be summarized in terms of the spin-spin correlation functions and the staggered magnetization, defined respectively as the ground-state expectation values,

$$
G^{\alpha \beta}(n) \equiv \frac{1}{s^{2}}\left\langle s_{k}^{\alpha} s_{k+n}^{\beta}\right\rangle ; \quad M^{\alpha} \equiv \frac{1}{s}\left|\left\langle s_{k}^{\alpha}\right\rangle\right| .
$$


Because of the translational invariance of the infinite chain, both $G^{\alpha \beta}(n)$ and $M^{\alpha}$ are independent of the index $k$ in Eq. (11). The asymptotic value of the magnitude of $G^{z z}(n)$ as $n \rightarrow \infty$ is equal to the square of the staggered magnetization $M^{z}$. The latter plays the role of the order parameter. It is equal to unity for the perfect Néel order of the Ising model.

In the critical region, $|\Delta| \leq 1$, there is no diagonal long-range order, i.e., $M^{z}=0$, and the leading asymptotes of the correlation functions have power-law decay behaviour as $n \rightarrow \infty,{ }^{38}$

$$
G^{z z}(n) \underset{n \rightarrow \infty}{\longrightarrow}\left\{\begin{array}{lr}
A_{1} n^{-2} ; & -1<\Delta \leq 0, \\
(-1)^{n} B_{1} n^{-\pi / \theta^{\prime}} ; & 0<\Delta \leq 1,
\end{array}\right.
$$

where $A_{1}$ and $B_{1}$ are constants which depend on $\Delta$, and the exponent $\theta^{\prime} \equiv \cos ^{-1}(-\Delta)$ lies in the range $\frac{\pi}{2}<\theta^{\prime} \leq \pi$. The critical regime thus has a "critical ferromagnetic" region for $-1<\Delta<0$ and a "critical antiferromagnetic" region for $0<\Delta<1$, separated by another phase boundary at $\Delta=0$.

In the Ising-like region, $\Delta>1$, the perfect Néel order of the Ising model has not been totally destroyed by the quantum fluctuations, and the long-range order is characterized by a nonzero value of the staggered magnetization, $M^{z}$. The spin-spin correlation function decays exponentially to a nonzero value,

$$
G^{z z}(n) \underset{n \rightarrow \infty}{\longrightarrow}(-1)^{n}\left[\mu+(\ell / \lambda)^{-n}\right] ; \quad \Delta>1,
$$

where $\mu=\left(M^{z}\right)^{2}$ and the correlation length $\lambda$ depend on $\Delta$. Very near the critical point $\Delta_{c}=1, M^{z}$ as a function of $\Delta$ shows the essential singularity behaviour, ${ }^{37}$

$$
M^{z} \underset{\Delta \rightarrow 1^{+}}{\rightarrow} \text { const } \times \sqrt{\Delta-1} \exp \left(-\frac{C_{1}}{\sqrt{\Delta-1}}\right),
$$

where $C_{1}$ is a positive constant.

The other main qualitative difference between the two antiferromagnetic phases concerns the presence or absence of a gap in the excitation spectrum. Thus, the excited states are generally described in terms of a dispersion law $\epsilon(k)$ for the excitation energy as a function of the momentum variable $k$ within the first Brillouin zone, namely $-\pi<k \leq \pi$ in 1D. Nevertheless, Faddeev and Takhtajan ${ }^{36}$ have shown that the fundamental excitation is that of a single $s_{T}=\frac{1}{2}$ kink with momentum $q$ such that $0 \leq q \leq \pi$. The exact excitation energy of this single-kink state in the critical phase is given as,

$$
\epsilon(q)=\frac{\pi}{2}\left(\frac{\sin \theta}{\theta}\right) \sin q ; \quad 0 \leq \theta \equiv \cos ^{-1} \Delta \leq \pi, \quad 0 \leq q \leq \pi .
$$

The spectrum of Eq. (15) has $\epsilon(0)=\epsilon(\pi)=0$, and is hence referred to as gapless. By contrast, in the Ising-like phase with nonvanishing long-range order, the single-kink state has excitation energy,

$$
\epsilon(q)=\frac{K_{1}}{\pi} \sinh \gamma\left(1-k_{1}^{2} \cos ^{2} q\right)^{\frac{1}{2}} ; \quad 1<\Delta \equiv \cosh \gamma,
$$

where the parameter $k_{1}$ is given in terms of the complete elliptic integrals $K_{1}$ and $K_{1}^{\prime}$,

$$
K_{1} \equiv \int_{0}^{\frac{\pi}{2}} d \theta\left(1-k_{1}^{2} \sin ^{2} \theta\right)^{-\frac{1}{2}} ; \quad K_{1}^{\prime} \equiv \int_{0}^{\frac{\pi}{2}} d \theta\left[1-\left(1-k_{1}^{2}\right) \sin ^{2} \theta\right]^{-\frac{1}{2}},
$$

by the relation

$$
\frac{K_{1}^{\prime}}{K_{1}}=\frac{\gamma}{\pi}
$$


We note that in this Ising-like phase, the excitation spectrum of Eq. (16) displays a nonzero gap at the zone boundary, $\epsilon(0)=\epsilon(\pi) \neq 0$.

We note finally that the observed physical excitations with $s_{T}^{z}= \pm 1$ are actually formed from two such kink solutions. The excitation energy obeys the exact additive relation,

$$
E_{k}(q)=\epsilon(k)+\epsilon(q-k),
$$

and the spectrum is thus a continuum with respect to the momentum parameter $k$, since the total momentum $q$ can take all values. The so-called antiferromagnetic spin waves $^{36}$ are precisely described by the lower boundary to this continuum.

The above $1 \mathrm{D}$ exact results all refer to the $s=\frac{1}{2}$ case. By contrast, the $s=1$ case in non-integrable. Prior to 1983, however, its general phase diagram was believed to be similar to that of the spin- $\frac{1}{2}$ case. Then, in 1983 , Haldane ${ }^{29}$ proposed the hitherto quite unexpected conjecture that there was a fundamental difference between the $s=n$ and $s=n+\frac{1}{2}$ cases, where $n$ is integral. In particular, he proposed for the $s=n$ case that the Ising-like antiferromagnetic phase with nonzero Néel order disappears as $\Delta$ decreases at some critical value $\Delta_{c}>1$, at which point the energy gap also disappears. Then, for $\Delta_{c}^{\prime}<\Delta<\Delta_{c}$, a new (Haldane) phase appears, with no counterpart in the spin- $\frac{1}{2}$ case. The Haldane phase has a non-degenerate ground state and is disordered. It has a nonzero energy gap in its spectrum and its spin-spin correlation functions decay exponentially to zero. In particular, the ground state of the isotropic Heisenberg model, $\Delta=1$, lies in the Haldane phase. Finally, for $\Delta<\Delta_{c}^{\prime}<1$, a gapless critical phase similar to that of the spin- $\frac{1}{2}$ case may also appear. Although not rigorously proven, the Haldane conjecture is by now strongly supported by Monte Carlo simulations, by exact diagonalizations for finite chains, and by various pieces of experimental data.

Let us now turn to the isotropic $(\Delta=1)$ Heisenberg antiferromagnet on lattices of higher dimensionality $d$, where rigorous results are still very scarce. Dyson, Lieb and $\operatorname{Simon}^{41}$ and later workers ${ }^{42}$ have managed to prove the strict presence of nonvanishing Néel order in the case where both $s \geq 1$ and $d \geq 2$. The most interesting spin- $\frac{1}{2}$ case is still highly controversial. The only exact result, apart, from the absence of Néel order for the $1 \mathrm{D}$ chain, concerns the $3 \mathrm{D}$ cubic lattice, for which Kennedy, Lieb and Shastry ${ }^{42}$ have proven the existence of nonzero Néel order. The $2 \mathrm{D}$ case remains of great interest but many open questions remain. ${ }^{43}$ For the (unfrustrated) square lattice there is now considerable consensus on the existence of long-range Néel order. ${ }^{27,28,44}$ For the 2D frustrated lattices the situation is far less clear. While the spin- $\frac{1}{2}$ Kagomé lattice Heisenberg antiferromagnet appears ${ }^{43}$ to be a candidate for a spin liquid of the type proposed by Anderson, ${ }^{45}$ rather than being Néel-like, the spin- $\frac{1}{2}$ triangular lattice remains controversial. In a very recent paper, Bernu et al. ${ }^{43}$ argue that the triangular Heisenberg antiferromagnet does possess nonzero Néel order. Although previous authors have reached the opposite conclusion, they present arguments as to why these previous analyses were flawed.

Returning to the spin- $\frac{1}{2} X X Z$ model on the square lattice, again few rigorous results are known despite strenuous efforts. Among the best available are those of Kubo and Kishi. ${ }^{46}$ These authors have applied various sum rules for the $X X Z$ model, and have shown that for the spin- $\frac{1}{2}$ square lattice the ground state possesses an off-diagonal longrange order akin to that of the planar (or $X Y$ ) state at small values of the anisotropy $(0<\Delta<0.13)$, and a diagonal long-range order of Néel type at large values of the anisotropy $(\Delta>1.78)$. Unfortunately, their proof cannot be extended to include the most interesting isotropic Heisenberg point at $\Delta=1$.

Although we have dwelt at length on the anisotropic Heisenberg (or $X X Z$ ) model, many other fascinating spin-lattice models exist, of which we mention just two, namely 
(i) the isotropic Heisenberg-biquadratic (HB) model; and (ii) the so-called $J_{1}-J_{2}$ or Majumdar-Ghosh (MG) ${ }^{47,48}$ model with isotropic nearest- and next-nearest-neighbour exchange. The corresponding Hamiltonians are given by,

$$
\begin{aligned}
& H_{H B}=\frac{1}{2} \sum_{\ell} \sum_{\rho}\left[\cos \omega\left(\mathbf{s}_{\ell} \cdot \mathbf{s}_{\ell+\rho}\right)+\sin \omega\left(\mathbf{s}_{\ell} \cdot \mathbf{s}_{\ell+\rho}\right)^{2}\right] ; \quad s \geq 1, \\
& H_{M G}=\frac{1}{2} \sum_{\ell}\left(\cos \omega \sum_{\rho} \mathbf{s}_{\ell} \cdot \mathbf{s}_{\ell+\rho}+\sin \omega \sum_{\delta} \mathbf{s}_{\ell} \cdot \mathbf{s}_{\ell+\delta}\right) ; \quad s \geq \frac{1}{2},
\end{aligned}
$$

where the sum over the (vector) index $\ell$ is over all $N$ spins with periodic boundary conditions, and the sums over the (vector) indices $\rho$ and $\delta$ respectively run over all nearest-neighbour and next-nearest-neighbour lattice vectors. Again, both models can be considered on arbitrary lattices, but we consider henceforth the available results only for the $1 \mathrm{D}$ chains. In both cases the zero-temperature phase diagrams are again rich in structure with respect to the parameter $\omega$.

The ground state of the spin-1 HB chain is ferromagnetic in the regime $5 \pi / 4>\omega>$ $\pi / 2$ and antiferromagnetic $\left(s_{T}^{z}=0\right)$ elsewhere, i.e., $-3 \pi / 4<\omega<\pi / 2$. The system is integrable using the Bethe ansatz $z^{32,35}$ at $\omega=-\pi / 4,{ }^{49}$ and at $\omega=+\pi / 4 .^{50}$ The exact ground state is also known at $\omega=-\pi / 2,{ }^{51}$ and at $\omega=\tan ^{-1}\left(\frac{1}{3}\right)$. In the latter case, Affleck et $a l^{42}$ showed that the system forms a valence bond solid antiferromagnet. The pure Heisenberg point, $\omega=0$, as discussed above was conjectured by Haldane ${ }^{29}$ to have a previously unexpected gap in its energy spectrum.

The spin-1 HB chain is now believed to have a doubly degenerate ground state and a gap in the entire region $-3 \pi / 4<\omega<-\pi / 4$. The ground state here is believed to be dimerized, with a spin-nematic phase also possibly present. ${ }^{52}$ At the integrable point $\omega=-\pi / 4$ a phase transition occurs to a Haldane phase with a nondegenerate ground state and a gap. This phase, which has twofold real-space periodicity, continues to exist for larger values of $\omega$ including the $\omega=\tan ^{-1}\left(\frac{1}{3}\right)$ point, probably right up to the integrable point $\omega=+\pi / 4$. The threefold real-space periodicity of the exact solution at $\omega=+\pi / 4$ is believed to exist over the whole of the region $\pi / 4 \leq \omega<\pi / 2$, and this trimerized phase appears to be gapless. ${ }^{53}$ Chubukov $^{52}$ predicts unusual ordering in both this region as well as in the dimerized regime for $-3 \pi / 4<\omega \lesssim-\pi / 2$.

We turn finally to the spin- $\frac{1}{2} \mathrm{MG}$ model in $1 \mathrm{D}$. In this case the model reduces to the isotropic Heisenberg antiferromagnet at $\omega=0$ and to two decoupled isotropic Heisenberg antiferromagnets at $\omega=\pi / 2$. Both points are hence exactly soluble by Bethe ansatz. ${ }^{32,35}$ Furthermore, at $\omega=\tan ^{-1}\left(\frac{1}{2}\right)$, the so-called $M G$ point, the exact ground state is a spin-Peierls state constructed from spontaneously dimerized uncorrelated spin-singlet nearest-neighbour pairs.

The zero-temperature phase diagram of the MG model exhibits (at least) three distinct regimes, namely (i) a ferromagnetic regime for $\pi-\tan ^{-1}\left(\frac{1}{4}\right)<\omega<3 \pi / 2$; (ii) an antiferromagnetic regime for $-\pi / 2<\omega<\tan ^{-1}\left(\frac{1}{2}\right)$; and (iii) a frustrated regime for $\tan ^{-1}\left(\frac{1}{2}\right)<\omega<\pi-\tan ^{-1}\left(\frac{1}{4}\right)$. Recent finite-chain studies ${ }^{54}$ have supported the view that the boundary between the two-fold spatially periodic antiferromagnetic regime and the frustrated regime occurs precisely at the $M G$ point, $\omega=\tan ^{-1}\left(\frac{1}{2}\right)$. There is also a phase transition within the antiferromagnetic regime at some value $\omega=\omega_{c}$ which separates a gapless spin-fluid state for $-\pi / 2<\omega<\omega_{c}$ from a dimerized state with a gap for $\omega_{c}<\omega<\tan ^{-1}\left(\frac{1}{2}\right)$. The position of this transition is given by Okamoto and Nomura ${ }^{55}$ as $\omega_{c} \approx \tan ^{-1}(0.2411)$. Since the system is known to have a gap and dimer-dimer long-range order at the $M G$ point $\omega=\tan ^{-1}\left(\frac{1}{2}\right)$, and to be a gapless spin 
liquid at $\omega=\pi / 2$, there is presumably also another phase transition in between these two values in the frustrated regime. Finally, the classical ground state in the frustrated regime (which has a slightly different boundary with the antiferromagnetic regime, namely at $\omega=\tan ^{-1}\left(\frac{1}{4}\right)$, than in the quantum-mechanical case) is a spiral structure whose real-space periodicity increases monotonically from two at the antiferromagnetic boundary to $N$, the total number of spins, at the ferromagnetic boundary. A detailed study of the correlation functions ${ }^{54}$ provides some evidence of similar behaviour in the quantum system.

While many more examples of quantum spin-lattice problems could be cited, the above examples should suffice to indicate the enormously rich, and in many cases still largely unexplored, phase structure that they exhibit.

\section{TYPICAL ELECTRON-LATTICE MODELS}

Having considered the previous spin-lattice models, it is natural to contemplate next the creation of a single or more than one vacancy on the lattice, and also to allow such vacancies to be dynamically mobile. Such classes of problems can no longer be described wholly in terms of the spin operators alone, and we must introduce instead the more basic electron creation and destruction operators, $c_{k \sigma}^{\dagger}$ and $c_{k \sigma}$ respectively, for an electron on lattice site $k$ with spin projection $\sigma\left(= \pm \frac{1}{2}\right)$. These operators obey the usual fermionic anticommutation relations,

$$
\left\{c_{k \sigma}, c_{k^{\prime} \sigma^{\prime}}^{\dagger}\right\}=\delta_{k k^{\prime}} \delta_{\sigma \sigma^{\prime}}
$$

An archetypal electron lattice Hamiltonian is that of the single-band Hubbard model, ${ }^{3}$

$$
H=-t \sum_{\langle i, j\rangle} \sum_{\sigma}\left(c_{i \sigma}^{\dagger} c_{j \sigma}+c_{j \sigma}^{\dagger} c_{i \sigma}\right)+U \sum_{i} n_{i \uparrow} n_{i \downarrow},
$$

where

$$
n_{i \sigma} \equiv c_{i \sigma}^{\dagger} c_{i \sigma}
$$

$t$ is the hopping matrix element which allows movement of electrons between the nearest-neighbour sites $\langle i, j\rangle$ without spin change, and $U$ is the on-site Coulomb repulsion term which acts to deter double occupancy of any site.

In the strong-coupling limit, $U \gg t$, the electrons prefer to occupy separate sites, and doubly occupied states are to be avoided. We may then perform standard strongcoupling perturbation theory or a simple canonical transformation to find the following effective Hamiltonian in this limit, ${ }^{55}$

$$
H=-t \sum_{\langle i, j\rangle} \sum_{\sigma}\left(\tilde{c}_{i \sigma}^{\dagger} \tilde{c}_{j \sigma}+\tilde{c}_{j \sigma}^{\dagger} \tilde{c}_{i \sigma}\right)+J \sum_{\langle i, j\rangle}\left(\mathbf{s}_{i} \cdot \mathbf{s}_{j}-\frac{\mathbf{1}}{4}\right)+(3 \text {-site terms })
$$

where

$$
\tilde{c}_{i \sigma} \equiv c_{i \sigma}\left(1-n_{i,-\sigma}\right) ; \quad J \equiv 4 t^{2} / U,
$$

and where, in a matrix notation,

$$
\mathrm{s}_{i} \equiv\left(c_{i \uparrow}^{\dagger} c_{i \downarrow}^{\dagger}\right) \frac{1}{2} \sigma\left(\begin{array}{c}
c_{i \uparrow} \\
c_{i \downarrow}
\end{array}\right)
$$

in terms of the usual Pauli matrices $\sigma \equiv\left(\sigma^{x}, \sigma^{y}, \sigma^{z}\right)$. When the (rather little studied) terms involving electron operators on three lattice sites are neglected, the effective Hamiltonian of Eq. (25) is just the well-known $t-J$ model. The first term in Eq. (25) 
now differs from its counterpart in Eq. (24) by the constraint implicit in the definition of Eq. (26) of no double occupancy of sites. Thus, the $t-J$ model has only three possible states per site, viz., an electron with spin up or down, or a hole. By contrast, the Hubbard model allows a fourth possible state per site, viz., double occupancy (i.e., by both an electron with spin up and an electron with spin down).

Although the Hubbard and $t-J$ models are equivalent to one another only for $t \ll U$ or for $t \gg J$, the $t-J$ model can also be studied in its own right for all values of $t / J$. Clearly, at precisely half-filling (i.e., $N$ electrons on $N$ lattice sites) the $t-J$ model is equivalent to the spin- $\frac{1}{2}$ isotropic Heisenberg model of Eq. (1), apart from the additive constant term which simply sets the energy scale so that the energy of a spin-1 electron pair $\langle i, j\rangle$ is zero. This reduction to the Heisenberg model is also easy to understand. Thus, this term is obtained from the large- $U$ limit of the Hubbard model in secondorder perturbation theory by summing over virtual transitions in which one electron first hops to a neighbouring site occupied by an electron of opposite spin, leaving a vacancy and a doubly occupied site, and then the two electrons on the doubly occupied site either revert to their original configuration or to one in which the two spins have been exchanged. This virtual transition is allowed only for nearest-neighbour antiparallel spins. The net effect is thus to shift the energy of a spin-zero electron pair downward with respect to an aligned (spin-one) pair. Since the Hubbard Hamiltonian is itself isotropic, the effective nearest-neighbour spin-spin interaction induced by this virtual transition is also isotropic, and the second (Heisenberg) term in Eq. (25) follows at once.

Many generalizations of the $t-J$ and Hubbard models have also been considered, especially in the context of models for the high-temperature superconductivity exhibited by the ceramic cuprates. For example, the inclusion of a next-nearest-neighbour hopping term $t^{\prime}$ leads to a similar $t-t^{\prime}-J$ model. Also, it is argued ${ }^{56}$ that a more realistic Hamiltonian for the electrons in the $\mathrm{CuO}_{2}$ planes is the so-called three-band Hubbard model. Zhang and Rice ${ }^{57}$ have also presented arguments for the similar reduction of the three-band Hubbard model to the $t$ - $J$ model as an effective Hamiltonian which faithfully captures the essential physics of relevance to the cuprate materials, although this view has been challenged by Emery and Reiter. ${ }^{58}$ For further details of these models the interested reader is referred to the recent reviews of $\mathrm{Lu} \mathrm{Yu}^{5}$ and Dagotto. ${ }^{59}$

Although the above electron lattice models have received a great deal of recent attention in connection with high-temperature superconductivity, many other interesting such models exist. As a single further illustration we cite the periodic Anderson model (PAM), which is a two-band model which has been used ${ }^{60}$ to describe the essential physics of such rare-earth mixed valence compounds as $\mathrm{SmB}_{6}$ and $\mathrm{CePd}_{3}$. These materials are characterized by a hybridization between the itinerant conduction electrons in a wide $d$-band and the strongly correlated localized electrons in a dispersionless $f$-band. The PAM Hamiltonian for a lattice with $N$ sites has the form,

$$
H=\sum_{\mathbf{k}, \sigma} \epsilon_{\mathbf{k}} c_{\mathbf{k} \sigma}^{\dagger} c_{\mathbf{k} \sigma}+E_{f} \sum_{i, \sigma} f_{i \sigma}^{\dagger} f_{i \sigma}+U \sum_{i} f_{i \uparrow}^{\dagger} f_{i \uparrow} f_{i \downarrow}^{\dagger} f_{i \downarrow}+N^{-\frac{1}{2}} V \sum_{\mathbf{k}, i, \sigma}\left(e^{i \mathbf{k} \cdot \mathbf{R} i} c_{\mathbf{k} \sigma}^{\dagger} f_{i \sigma}+\text { h.c. }\right),
$$

where $f_{i \sigma}$ and $c_{\mathrm{k} \sigma}^{\dagger}$ are respectively the creation operators for an electron with spin $\sigma$ at the lattice site $i$ with position vector $\mathbf{R}_{i}$ in the $f$-band, and a conduction electron with spin $\sigma$ and lattice momentum k. The energy of the $f$-orbital is $E_{f}$ and the conduction band has energy dispersion $\epsilon_{\mathbf{k}}$. For example, in units where the conduction bandwidth is set to unity and the energy zero is at the bottom of the conduction band, the 1D chain has $\epsilon_{k}=\frac{1}{2}(1-\cos k) ;-\pi<k \leq \pi$. Finally, the parameter $V$ measures the hybridization between the two bands, and $U$ is the on-site Coulomb repulsion term 
which deters double occupancy of the $f$-orbitals.

With very few exceptions the only exact results known for electron lattice models are for 1D chains. Such systems are not without experimental interest, in view of their relevance to various quasi-1D systems such as conducting polymers ${ }^{61}$ and organic conductors. ${ }^{62}$ Models such as the 1D Hubbard model have been solved by Bethe ansatz techniques. ${ }^{63}$ Furthermore, Haldane ${ }^{64}$ has stressed the important relationship of such exactly soluble 1D lattice models solved by Bethe ansatz techniques with various 1D continuous models of the Luttinger type ${ }^{65}$ which have been solved by renormalization group and bosonization techniques (and see Ref. 5 for a recent overview). This work has led to the realization that there is a universality class of so-called Luttinger liquids, to which several of the electron-lattice models soluble by Bethe ansatz belong, with very different properties from the more familiar Fermi liquids of Landau, and whose properties can be mapped onto the Fermi gas. Much recent work has gone into the study of such Luttinger liquids, particularly with regard to investigating their relevance to the study of high-temperature superconductivity.

\section{HAMILTONIAN LATTICE GAUGE MODELS}

The quantum chromodynamics (QCD) of interacting quarks and gluons is widely accepted as being the best available theory of the strong interaction. A full derivation of the hadronic spectrum and a demonstration of quark confinement from QCD therefore requires a careful treatment of this non-Abelian gauge field theory in the nonperturba-

tive sector. A common approach to this end is via the lattice formulation, ${ }^{66,67}$ which introduces an ultraviolet cutoff that makes the theory well-defined as well as strictly preserving the important local gauge invariance. ${ }^{68}$

Since non-Abelian theories are technically more complicated than Abelian ones, it has been natural to study the latter first. In particular, both the continuous compact gauge group $U(1)$ describing conventional quantum electrodynamics (QED), ${ }^{67,69-71}$ and the discrete gauge groups $\{Z(N) ; N=2,3, \cdots\}^{72,73}$ discussed below, have been widely studied on the lattice. The latter groups are simply the sets of complex $N$ th roots of unity, with ordinary multiplication as the group operation. They are interesting for at least three important reasons. Firstly, as $N \rightarrow \infty$, the group $Z(N)$ passes smoothly over to $U(1)$. Secondly, for $N=2$, the $Z(2)$ lattice gauge theory reduces to a gaugeinvariant generalization of the Ising model, which was first considered by Wegner ${ }^{74}$ as an example of a condensed matter system exhibiting a phase transition but without possessing a local order parameter. Thirdly, the $Z(N)$ gauge theories are also closely connected to the spin-glass models of Edwards and Anderson. ${ }^{75}$

Migdal $^{76}$ first noted that the close connection of the $Z(2)$ gauge model to the Ising spin model is but one example of a more general and rather remarkable set of such relationships. Thus, many $(3+1)$-dimensional gauge field theories have a counterpart in some (1+1)-dimensional spin-chain, in a number of well-defined senses, as described in some detail, for example, by Fradkin and Susskind. ${ }^{70}$ The principal difference between such spin models as the Ising models and such gauge field models as $Z(2)$ lies in the incorporation in the latter at a local level of a symmetry that is present only globally in the former. Thus, whereas the Ising model is globally symmetric under the interchange of the direction of all the spins, in the $Z(2)$ model this symmetry is incorporated locally with respect to the interchange of only those spins (now defined on the links of the lattice) connected to a given site, as we see in more detail below. The common feature of all gauge symmetries ${ }^{66,67,72,74}$ is this invariance with respect to an operation affecting only degrees of freedom localized to some site on the lattice. Furthermore, 
the imposition of a gauge symmetry hugely restricts the possible Hamiltonians.

In the Hamiltonian formulation of $(d+1)$-dimensional lattice gauge theory, ${ }^{67}$ one works on a $d$-dimensional hypercubic lattice with lattice spacing $a$ in discretized space, and allows time to remain continuous. The fundamental entities are the group elements $U_{\ell}$, defined on the links $\{\ell\}$ of the lattice in terms of the group generators. For example, in the $S U(N)$ case, the basic group generators are the $N \times N$ matrices $\left\{\frac{1}{2} \lambda^{a} ; a=\right.$ $\left.1,2, \cdots, N^{2}-1\right\}$ which obey the usual group commutation relations. We define a corresponding set of "chromoelectric" fields $\left\{E_{\ell}^{a}\right\}$ with $N^{2}-1$ components. In the axial or temporal gauge we also define a set of chromo-vector potentials $\left\{A_{\ell}^{a}\right\}$, such that

$$
U_{\ell}=\exp \left(i \frac{1}{2} \sum_{a} \lambda^{a} A_{\ell}^{a}\right) .
$$

The canonical commutation relations that quantize the theory are,

$$
\left[E_{\ell}^{a},\left(U_{\ell^{\prime}}\right)_{i j}\right]=\delta_{\ell \ell^{\prime}} \sum_{k=1}^{N}\left(\frac{1}{2} \lambda^{a}\right)_{i k}\left(U_{\ell}\right)_{k j} ; i, j=1, \cdots, N .
$$

For example, in the case of $U(1)$, where $\frac{1}{2} \lambda^{a} \rightarrow 1$, we simply have,

$$
U_{\ell}=\mathrm{e}^{i A_{\ell}} ; \quad-\pi<A_{\ell} \leq \pi,
$$

and Eq. (30) is equivalent to the usual canonical commutation relation,

$$
\left[E_{\ell}, A_{\ell^{\prime}}\right]=-i \delta_{\ell \ell^{\prime}}
$$

Similarly, in the case of $S U(2)$, where $\lambda^{a} \rightarrow \sigma^{a}$, the Pauli matrices, we have

$$
\begin{aligned}
U_{\ell} & =\exp \left(\frac{1}{2} i \phi_{\ell} \hat{\mathbf{n}}_{\ell} \cdot \boldsymbol{\sigma}\right) \\
& =\cos \left(\frac{1}{2} \phi_{\ell}\right) \mathbf{1}+i \sin \left(\frac{1}{2} \phi_{\ell}\right) \hat{\mathbf{n}}_{\ell} \cdot \boldsymbol{\sigma} ; \quad 0<\phi_{\ell} \leq 2 \pi,
\end{aligned}
$$

where $\hat{\mathbf{n}}_{\ell}$ is a unit vector.

Just as in the usual continuum QED the field $\mathbf{A}(\mathbf{x})$ is not a gauge-invariant quantity, so on the lattice nor is its link counterpart $A_{\ell}^{a}$. The gauge-invariant quantities associated with $A_{\ell}^{a}$ or $U_{\ell}$ simply arise from considering closed loops on the lattice. In particular, the simplest such loop is the (elementary square) plaquette formed from four links, say $\ell=$ $1,2,3,4$ in cyclic order. We thereby define a group-element-valued plaquette variable $U_{p}$ as an oriented product of the link group elements taken around the plaquette,

$$
U_{p} \equiv U_{1} U_{2} U_{3}^{\dagger} U_{4}^{\dagger}
$$

The $(d+1)$-dimensional $S U(N)$ Kogut-Susskind ${ }^{67}$ Hamiltonian in the vacuum or pure gauge sector (without fermions, i.e., the photonic or charge-free sector for $U(1)$, or the gluonic or quark-free sector for $S U(N)$, particularly $N=3$ ) is given as,

$$
\begin{gathered}
W=g^{2} a^{2-d} H, \\
H=\frac{1}{2} \sum_{\ell} \sum_{a} E_{\ell}^{a} E_{\ell}^{a}+\lambda \sum_{p}\left(N-\operatorname{Re} \operatorname{Tr} U_{p}\right),
\end{gathered}
$$

where $\lambda \equiv 2 a^{2 d-6} / g^{4}$ is the dimensionless coupling constant which approaches infinity in the physical continuum limit. 
Clearly, in the $U(1)$ case we have the simple relation,

$$
U_{p}=\mathrm{e}^{i B p} ; \quad B_{p}=A_{1}+A_{2}-A_{3}-A_{4}
$$

which is just the lattice equivalent of the usual relationship for the magnetic field, $\mathbf{B}=\nabla \times \mathbf{A}$, for the plaquette formed from the links $1,2,3,4$ in cyclic order. The $U(1)$ Hamiltonian thus becomes,

$$
H=\frac{1}{2} \sum_{\ell} E_{\ell}^{2}+\lambda \sum_{p}\left(1-\cos B_{p}\right)
$$

where the periodicity in the magnetic term plays a crucial role in maintaining the true phase structure and gauge-invariance properties of the theory. ${ }^{69}$ In the continuum limit, Eq. (37) reduces to the usual $U(1)$ Hamiltonian (for the pure electromagnetic field, for example),

$$
H \rightarrow \frac{1}{2} \int d^{d} x\left[\mathbf{E}^{2}(\mathbf{x})+\mathbf{B}^{2}(\mathbf{x})\right] .
$$

We also observe that the Hamiltonian $H$ of Eq. (37) is locally gauge-invariant if at some given site $n$ on the lattice we rotate the phases $A_{\ell}, \ell \ni n$, by a set amount and $H$ is thereby unchanged. More precisely, these $U(1)$ gauge transformations are generated by the operators,

$$
G\left[\omega_{n}\right] \equiv \exp \left(i \sum_{n} \omega_{n} D_{n}\right),
$$

where $D_{n}$ is the lattice equivalent of $\mathbf{D} \equiv \boldsymbol{\nabla} \cdot \mathbf{E}$, namely,

$$
D_{n} \equiv \sum_{\ell \ni n} E_{\ell}
$$

for the oriented variables $E_{\ell}$. The mode of action of the generators $G\left[\omega_{n}\right]$ on the basic variables $A_{\ell}$ and $E_{\ell}$ defined on a link $\ell$ joining the nodes $n$ and $n^{\prime}$, and on the plaquette variable $B_{p}$, is easily seen to be given by

$$
\begin{gathered}
A_{\ell}^{\prime} \equiv G^{-1} A_{\ell} G=A_{\ell}+\omega_{n^{\prime}}-\omega_{n}, \\
E_{\ell}^{\prime} \equiv G^{-1} E_{\ell} G=E_{\ell} \\
B_{p}^{\prime} \equiv G^{-1} B_{p} G=B_{p}
\end{gathered}
$$

and hence the Hamiltonian is gauge-invariant, $G^{-1} H G=G$. We also note that a wave function $|\psi\rangle$ is left invariant by $G$, namely $G|\psi\rangle=|\psi\rangle$ only if $D_{n}|\psi\rangle=0, \forall n$. By comparison with Gauss' law, this clearly implies that $|\psi\rangle$ belongs to the uncharged sector where none of the nodes carries charge, which is compatible with the Hamiltonian itself describing only the pure gauge field sector.

The further reduction of Eq. (37) to a standard problem in quantum many-body theory in terms of the compact variables $\left\{B_{p} ;-\pi<B_{p} \leq \pi\right\}$ alone is now straightforward. Since it is described in detail elsewhere in this volume, ${ }^{77}$ we do not repeat it here. Instead, we turn our attention to the $Z(N)$ models. The corresponding link variables $\left\{P_{\ell}, Q_{\ell}\right\}$ are now defined to obey the $Z(N)$ algebra,

$$
\begin{aligned}
P_{\ell}^{N} & =Q_{\ell}^{N}=1, \quad P_{\ell}^{\dagger} P_{\ell}=Q_{\ell}^{\dagger} Q_{\ell}=1, \\
P_{\ell} Q_{\ell} & =\exp (-2 \pi i / N) Q_{\ell} P_{\ell} .
\end{aligned}
$$


Operators on different links commute. Equation (42) implies that $P_{\ell}$ and $Q_{\ell}$ are ladder operators with respect to each other. For example, in a representation in which $P_{\ell}$ is diagonalized,

$$
P_{\ell}|L\rangle=\exp (-2 \pi i L / N)|L\rangle,
$$

the operator $Q_{\ell}$ has the mode of action,

$$
Q_{\ell}|L\rangle=|L+1\rangle ; \quad|L+N\rangle=|L\rangle .
$$

Equivalently, each link $\ell$ has associated with it an element $U_{\ell}$ of the group $Z(N)=$ $\left\{\mathrm{e}^{i n \delta} ; \delta=2 \pi / N, n=1,2, \cdots, N\right\}$. Clearly, for the case $N=2$, the algebra of Eq. (42) can be realized by choosing $P_{\ell}$ and $Q_{\ell}$ to be any two of the usual Pauli matrices, $\left\{\sigma_{i}(\ell) ; i=1,2,3\right\}$, defined on the links $\{\ell\}$. We make here the standard choice of $\sigma_{1}(\ell)$ and $\sigma_{3}(\ell)$.

The quantum $Z(N)$ Hamiltonian is taken to be ${ }^{70,73,78}$

$$
H=\frac{1}{2} \sum_{\ell}\left(2-P_{\ell}^{\dagger}-P_{\ell}\right)-\frac{1}{2} \lambda \sum_{p}\left(Q_{1} Q_{2} Q_{3}^{\dagger} Q_{4}^{\dagger}+\text { h.c. }-2\right),
$$

where the second term involves a sum over plaquettes $\{p\}$ bounded by the links $\ell=(1,2,3,4)$ in cyclic order. The $N \rightarrow \infty$ limit is readily related ${ }^{73}$ to the $U(1)$ model above, after a simple re-scaling of the first term and by making the following representations of the operators $P_{\ell}$ and $Q_{\ell}$,

$$
P_{\ell}=\exp \left(-\frac{2 \pi i}{N} E_{\ell}\right) ; \quad Q_{\ell}=\exp \left(i A_{\ell}\right),
$$

where $E_{\ell}$ and $A_{\ell}$ are dimensionless canonically conjugate Hermitian operators obeying the commutation relation of Eq. (32).

After the omission of some trivial constant terms, the Hamiltonian of Eq. (45) can be rewritten for the $Z(2)$ model in the form,

$$
H=-\sum_{\ell} \sigma_{1}(\ell)-\lambda \sum_{p} \sigma_{3}(1) \sigma_{3}(2) \sigma_{3}(3) \sigma_{3}(4) .
$$

We may define a local unitary gauge operator $G(n)$ analogous to Eq. (39) for the $U(1)$ model as,

$$
G(n)=\prod_{\ell \ni n} \sigma_{1}(\ell)
$$

where the product runs over all $2 d$ links attached to the site $n$, for a model in $d$ spatial dimensions. The mode of action of $G(n)$ on the spin operators is as follows,

$$
\begin{aligned}
& G^{-1}(n) \sigma_{1}(\ell) G(n)=\sigma_{1}(\ell) ; \quad \forall \ell, \\
& G^{-1}(n) \sigma_{3}(\ell) G(n)= \begin{cases}-\sigma_{3}(\ell) ; & \ell \ni n, \\
+\sigma_{3}(\ell) ; & \ell \not \supset n .\end{cases}
\end{aligned}
$$

Clearly, all operators $G(n)$ commute with the Hamiltonian of Eq. (47).

The ground state $|\Psi\rangle$ of the $Z(2)$ Hamiltonian may also be proven ${ }^{68}$ to be gaugeinvariant under the action of $G(n)$, so that $G(n)|\Psi\rangle=|\Psi\rangle, \forall n$. Clearly, one has immediately that the expectation value of the operator $\sigma_{3}(\ell)$ in any gauge-invariant state must vanish identically. Hence, there can be no nonzero magnetization in any state satisfying gauge invariance, and no phase transition in any $Z(2)$ model can lead to a magnetized phase. Nevertheless, as was first pointed out by Wegner ${ }^{74}$ the $Z(2)$ 
model for various values of the spatial dimensionality $d$ does exhibit phase transitions as outlined below. Thus, the lattice spin models with a local gauge symmetry have very different phase structure and order parameters from the models discussed in the second Section. The situation discussed above is actually a particular example of Elitzur's theorem $^{68}$ that a local symmetry cannot break down spontaneously. By contrast, in the Ising model, for example, one has a doubly degenerate ground state in which the global symmetry transformation of reversing all of the spins takes one degenerate state to the other.

Finally, we note that some exact relations are known for the lattice gauge models in $(d+1)$ dimensions. For example, certain well-defined duality relations are known for the $Z(2)$ model. ${ }^{70,72-74}$ In two space dimensions $(d=2)$ the theory is dual to the Ising model in two spatial dimensions in a transverse magnetic field, and is thus a two-phase system with a second-order phase transition at some critical coupling, $\lambda=\lambda_{c}$. It is also known that for $d=1$ (i.e., a $1 \mathrm{D}$ chain of plaquettes), gauge theory is exactly solvable. ${ }^{72,79}$ For an up-to-date review of these and other exact results, the reader is referred to the excellent recent book by Montvay and Münster. ${ }^{80}$

\section{QUANTITATIVE MICROSCOPIC MANY-BODY TECHNIQUES FOR LATTICE HAMILTONIANS}

The traditional means for dealing with lattice Hamiltonian systems of the sort described in the previous three Sections have largely relied on the following methods or their close variants: (i) various Monte Carlo methods (e.g., of the variational, Green's function, diffusion, or path-integral varieties); (ii) exact diagonalizations on small lattices (using, e.g., the Lanczos technique); (iii) variational estimates; and (iv) perturbation theory in the strong- and/or weak-coupling limits, followed by some (generalized) Padé or similar re-summation technique to continue the series outside the perturbative regime (i.e., in general, beyond its radius of convergence if one exists). Both methods (i) and (ii) suffer from the problem that they are restricted to finite lattices, and one needs to extrapolate the results to the infinite lattice of interest. This can be highly nontrivial, especially for the exact diagonalizations, where for systems of higher dimensionality in particular, the spatial extent of the lattices calculable with even the largest computers available remains rather small. One can thus easily miss the presence of long-range correlations or subtle ordering properties, especially near the phase transitions which are often of prime interest.

The Monte Carlo studies are almost always most successful when the Hamiltonian can be mapped onto an equivalent bosonic problem, or where all off-diagonal terms in it can be made positive- (or negative-) definite with a suitable choice of basis. In this way one may circumvent the infamous "minus sign problem" which makes Monte Carlo studies of dynamical fermion systems so difficult. Thus, for example, whereas Monte Carlo studies have been extremely successful for the spin- $\frac{1}{2}$ Heisenberg model on a $2 \mathrm{D}$ square lattice, ${ }^{27,28,44}$ similarly accurate studies of the analogous Hubbard and $t-J$ models involving vacancies on the lattice still pose a very severe challenge for Monte Carlo algorithms.

For many lattice systems huge efforts have been expended to get high-order terms in a perturbation series, typically also involving large amounts of supercomputer time. Commonly, 10-20 (or more) terms are known. Nevertheless, the further analysis to resum or to extend the range of validity of such series is often extremely difficult. A typical recent attempt for the $U(1)$ lattice gauge model in $(2+1)$ dimensions, ${ }^{81}$ for example, starts from the strong-coupling $(\lambda \rightarrow 0)$ perturbation series for the Hamiltonian of 
Eq. (37). It utilizes the known coefficients up to $O\left(\lambda^{18}\right)$, as well as the known weakcoupling $(\lambda \rightarrow \infty)$ limit, as input to generalized Padé approximants. Even this very careful analysis, however, loses essentially all accuracy for even the ground-state energy, for $\lambda \gtrsim 5$.

Similarly, while variational calculations are often an extremely valuable aid in the conceptualization of particular models, they can provide very deceptive results for properties such as order parameters or for correlation functions, even when their estimates for the energy eigenvalues are good. A typical example is a calculation by Liang, Doucot and Anderson ${ }^{82}$ for the spin- $\frac{1}{2}$ Heisenberg model on the $2 \mathrm{D}$ square lattice employing a variational wave function including long-range resonating valence bonds. This trial wave function gives a ground-state energy per spin of $-0.6688 \pm 0.0004$ from a variational Monte Carlo estimate, which is very close to the currently best estimate of -0.6696 . Nevertheless, the trial wave function gives a vanishing staggered magnetization, compared to the current best estimate of about $60 \%$ of the Néel value. Indeed, Liang et al. originally argued that the $2 \mathrm{D}$ spin- $\frac{1}{2}$ Heisenberg antiferromagnet on the square lattice was close to criticality on the basis that their energy estimate was so close to the exact result. Clearly, such claims are difficult to sustain both in theory and in practice. Such optimized variational wave functions are often also used in diffusion or Green's function Monte Carlo simulations, for example, as the trial functions used for the importance sampling which usually needs to be introduced to reduce the statistical error. One must be aware that their use here can also introduce unknown systematic errors beyond the cited statistical errors.

Other nonperturbative techniques that have also been less extensively used for lattice Hamiltonians include, for example, the block renormalization group method, and the $t$-expansion (or projector expansion) method. ${ }^{83}$ The latter method is somewhat analogous to the high-temperature series expansion in statistical mechanics. It uses the operator $\exp (-H t)$ to project trial states onto the low-lying eigenstates of the Hamiltonian $H$, after which expectation values are estimated by means of a power series expansion followed by a suitable analysis of the series to re-sum or extend it.

In view of the important role that lattice Hamiltonians are clearly now playing both theoretically and practically, the application to them of other ab initio techniques from microscopic quantum many-body theory appears to be timely. Furthermore, this is a field in which enormous progress has been made over the last decade or more. Thus, quantum many-body theory has both deepened at the level of formal developments and broadened in its range of successful applications. Two methods in particular have attracted special interest because of their demonstrated versatility, their ability to achieve very high accuracy at attainable levels of approximation, and their capacity for systematic improvement. These are the correlated basis function (CBF) method ${ }^{16-23}$ and the coupled cluster method (CCM) ${ }^{6-15}$ upon which we focus in the remainder of this Section. These two methods are nowadays widely recognized as providing the most powerful microscopic formulations currently available for dealing at an $a b$ initio level with fundamental quantum many-body problems.

It is also pertinent in this context to note that strong pessimism and reservations have been voiced by Wilson, concerning the developments still needed within lattice QCD before meaningful comparisons with experiment can occur. Thus, in a muchcited 1990 paper, ${ }^{84}$ written some 15 years after he founded lattice gauge theory, ${ }^{66}$ Wilson remarks that "Lattice gauge theory is still in its very early stages. It does not have the computing power it needs to produce results useful to experimentalists, nor has it yet had the algorithmic advances that quantum chemistry has undergone." He also states that "I still believe that extraordinary increase in computing power $\left(10^{8}\right.$ is I think 
not enough) and equally powerful algorithmic advances will be necessary before a full interaction with experiment takes place." Wilson further recommends the lattice gauge theory community to look especially to the field of quantum chemistry for new ideas and inspiration, particularly since both fields have a shared concern with many-fermion systems interacting via long-range (unscreened) forces. Our own view is that in this context Wilson could, equally validly, have extended his recommendation to include the field of quantum many-body theory in general, and the CCM in particular.

The very high accuracy required, for example, for the calculation of parity violation in atoms or molecular energy differences of chemical significance, requires extremely accurate treatments of the electronic correlations. The CCM is ideally suited for such applications ${ }^{85}$ where it is widely regarded as the method of first choice in terms of power and accuracy. Typical calculations involve ionization potentials, electron affinities, Auger spectroscopy, excitation energies, and energy gradients to use in the search of potential energy surfaces to predict vibrational spectra or to locate transition states in decomposition reactions. Many atoms and molecules have been so studied with the CCM, with state-of-the-art calculations involving molecules with up to about eighty active electrons.

We now give a very brief overview of both the CBF method and the CCM.

\section{The Correlated Basis Function (CBF) Method}

The most common, and perhaps the simplest, of the variational methods in quantum many-body theory is based on a trial wave function of the (Bijl-Dingle-) Jastrow type. ${ }^{86}$ Early calculations of this sort relied on various cluster expansions of the ensuing approximate matrix elements. ${ }^{86-88}$ It was realized later that these variational approaches may also be formulated diagrammatically. ${ }^{89}$ This feature has been of considerable help in the construction of such powerful approximations as the Percus-Yevick and hypernetted chain (HNC) summations and their variants, which have their origins in the classical theory of liquids and which have been adapted for both bosonic and fermionic systems. ${ }^{16,90}$ The review article by Clark $^{91}$ gives a good overview of the variational theory sketched above as applied to extended nuclear matter. The interested reader is also referred to Ref. 92 .

Two basic flaws mar the above variational approaches. Firstly, the particular partial summations of the graphs considered by such approximations as the HNC approach destroy one of the most attractive features of the variational techniques, namely that they yield upper bounds to the exact ground-state energy. Secondly, even a complete summation of graphs (or a variational Monte Carlo evaluation of the corresponding expectation values) for a given trial wave function (of Jastrow type, for example) gives only the exact variational result and not the true ground state. This latter deficiency may be remedied by the inclusion of more general state-dependent correlations and higher-order correlation functions of the Feenberg type. Alternatively, and more generally, one may extend the Jastrow wave function to a complete set of correlated basis functions (CBF).

The CBF method was introduced some 35 years ago by Feenberg and his collaborators, ${ }^{16,17}$ and was later developed largely by Clark and his collaborators. ${ }^{18-23}$ The present author has given in an earlier volume of this series a very brief qualitative survey of the method, ${ }^{93(a)}$ which contains many citations of the original literature; and a more detailed recent introductory survey is given in Ref. 93(b). The CBF method has as its central ingredient the direct incorporation of the most important interparticle correlations into the approximate wave functions on which the microscopic description 
is based. At it simplest level the method involves only a single configuration, and hence reduces to ordinary variational theory. This further reduces to Jastrow theory if the simplest reasonable choice of correlation operator is made in terms of the usual symmetric product over all pairs in the system of state-independent two-body correlation functions.

At its most general level, the CBF method constructs for an $N$-body system a multiconfigurational correlated basis $\left\{\left|\Psi_{I}\right\rangle\right\}$ of normalized but generally nonorthogonal state vectors,

$$
\left|\Psi_{I}\right\rangle=\frac{F\left|\Phi_{I}\right\rangle}{\left\langle\Phi_{I}\left|F^{\dagger} F\right| \Phi_{I}\right\rangle^{1 / 2}},
$$

in terms of a correlation operator $F$ applied to a complete orthonormal basis $\left\{\left|\Phi_{I}\right\rangle\right\}$ of model states. The latter usually carry the correct quantum statistics and any essential symmetries of the system. They might, for example, be chosen to provide an adequate lowest-order description of the system if it were not strongly interacting. For an application to an electronic system, for example, the states $\left\{\left|\Phi_{I}\right\rangle\right\}$ could thus be chosen as a set of Slater determinants of some appropriate single-electron orbitals. However, as alternatives to the resulting Hartree-Fock or filled Fermi sea state for the model ground state $\left|\Phi_{0}\right\rangle$, for example, one could also choose a BCS state, a spin- or charge-density wave state, etc., depending on the problem under consideration.

With the above considerations in mind, the correlation operator $F$ is thus usually symmetric in the complete sets of single-particle quantum numbers used to denote the $N$ particles. Most importantly, $F$ also possesses the cluster decomposition property, namely that upon spatial separation of one subgroup of particles (say, $1,2, \cdots, n)$ far from the remainder $(n+1, n+2, \cdots, N)$, the operator $F(1,2, \cdots, N)$ decomposes into a product,

$$
F(1,2, \cdots, N) \rightarrow F^{(n)}(1,2, \cdots, n) F^{(N-n)}(n+1, n+2, \cdots, N) .
$$

It is this property which allows the natural definition of correlation operators $F^{(n)}(1,2, \cdots, n)$ for $n$-body subsystems, with $1 \leq n \leq N$, in terms of a given $N$ body operator, $F \equiv F^{(N)}$. It is also the key ingredient in allowing a linked-cluster expansion for physical quantities.

Just as the CBF method allows considerable freedom in the choice of model states $\left\{\left|\Phi_{I}\right\rangle\right\}$, so too is there great flexibility in the parametrization of $F$. For example, the simplest choice is presumably the state-independent Jastrow form, $F \rightarrow F_{J}$, where $F_{J}$ may be specified in its usual coordinate-space form in terms of the relative separation $r_{i j}$ between particles $i$ and $j$ as,

$$
F_{J}=\prod_{1 \leq i<j \leq N} f\left(r_{i j}\right)
$$

The more general Feenberg form, $F \rightarrow F_{F}$, is given in terms of higher-order correlation functions as,

$$
F_{F}=\prod_{i<j} f_{2}\left(r_{i j}\right) \prod_{k<\ell<m} f_{3}\left(r_{k \ell}, r_{k m}, r_{\ell m}\right) \cdots .
$$

It is also possible to introduce state-dependent two-body (and, more generally, $n$-body) correlations. Physical examples include velocity-dependent correlations to include backflow effects, or a spin-dependent term such as,

$$
F_{\mathrm{ss}}=\mathcal{S} \prod_{i<j}\left[1+\eta\left(r_{i j}\right) \mathbf{s}_{i} \cdot \mathbf{s}_{j}\right],
$$

to include spin-spin correlations, etc. 
Once a choice for the form of $F$ has been made, the CBF method devolves onto the computation of the matrix elements $H_{I J}$ and $N_{I J}$ of the Hamiltonian $H$ and the unit operator respectively,

$$
H_{I J} \equiv\left\langle\Phi_{I}\left|F^{\dagger} H F\right| \Phi_{J}\right\rangle ; \quad N_{I J} \equiv\left\langle\Phi_{I}\left|F^{\dagger} F\right| \Phi_{J}\right\rangle .
$$

One assumes that any exact stationary energy eigenvector $|\Xi\rangle$, such that $H|\Xi\rangle=E|\Xi\rangle$, may now be (approximately) expanded in the correlated multiconfigurational basis as follows,

$$
|\Xi\rangle=\sum_{J} C_{J}\left|\Psi_{J}\right\rangle
$$

The Schrödinger equation is decomposed into the coupled set of linear generalized eigenvalue equations,

$$
\sum_{J}\left(H_{I J}-E N_{I J}\right) C_{J}=0
$$

with the resulting nontrivial solution for the coefficientss $\left\{C_{I}\right\}$ if and only if $E$ satisfies the secular equation,

$$
\operatorname{det}\left(H_{I J}-E N_{I J}\right)=0 .
$$

The heart of the CBF method lies in the accurate numerical evaluation of the manybody matrix elements $\left\{H_{I J}\right\}$ and $\left\{N_{I J}\right\}$. Various ways to do this all fall within the purview of CBF theory. For example, various cluster-expansion techniques ${ }^{88,94}$ have been developed, which also provided a basis for much of the later diagrammatic analysis of both diagonal elements (expectation values) ${ }^{16,89,90}$ and off-diagonal elements. ${ }^{19}$ This latter work further led to the definition of the CBF effective interaction and to many illuminating connections with conventional diagrammatic many-body perturbation theory. Exploration of these connections has allowed techniques from standard time-independent many-body perturbation theory, as developed for weakly interacting systems, to be modified into their CBF counterparts for use with strongly interacting systems. ${ }^{22}$

Ultimately, however the matrix elements $\left\{H_{I J}\right\}$ and $\left\{N_{I J}\right\}$ are derived, the generalized eigenvalue problem of Eqs. (57) and (58) must be solved in some approximate, heuristic, or systematic manner. Various such methods have been used in this context. They include nonorthogonal perturbation theory $;^{18}$ straightforward diagonalization of the configuration-interaction method type (i.e., in the space spanned by some subset of the full correlated basis) $;^{18,95}$ and a correlated version of the random phase approximation (RPA), which treats semiclassically the small-amplitude oscillations about an equilibrium correlated CBF configuration. ${ }^{21-23}$

\section{The Coupled Cluster Method (CCM)}

A quantitative description of the multiparticle correlations in a quantum manybody system requires a model (or reference) state $|\Phi\rangle$ for comparison purposes. The principal requirement on $|\Phi\rangle$ for a CCM formulation is that it forms a cyclic vector with respect to which we may define two Abelian subalgebras. One is formed from the multiconfigurational creation operators $\left\{C_{J}^{\dagger}\right\}$, with $\left[C_{J}^{\dagger}, C_{J^{\prime}}^{\dagger}\right]=0$; and the other from their Hermitian-conjugate destruction counterparts. Each set-index $J$ here labels a general multiparticle cluster configuration with respect to $|\Phi\rangle$, which thus plays the role of vacuum or reference state. We require that the operators $\left\{C_{J}^{\dagger}\right\}$ and $\left\{C_{J}\right\}$ are complete, in the sense that arbitrary ket and bra states within the appropriate manybody Hilbert space $\mathcal{H}$ may be decomposed as,

$$
|\Psi\rangle=\sum_{J} f_{J} C_{J}^{\dagger}|\Phi\rangle ; \quad\langle\tilde{\Psi}|=\sum_{J} \tilde{f}_{J}\langle\Phi| C_{J}
$$


This is clearly achievable if the identity operator $I$ within $\mathcal{H}$ has the resolution,

$$
I=\sum_{J} C_{J}^{\dagger}|\Phi\rangle\langle\Phi| C_{J}
$$

It is also convenient, but not essential, in practice if the operators form an orthonormal set,

$$
\left\langle\Phi\left|C_{J} C_{J^{\prime}}^{\dagger}\right| \Phi\right\rangle=\delta\left(J, J^{\prime}\right)
$$

where $\delta\left(J, J^{\prime}\right)$ is some suitably defined (generalized) delta function between the setindices $\{J\}$.

As an illustration we consider a system of $N$ electrons. The state $|\Phi\rangle$ may then be chosen as an $N$-electron Slater determinant formed from the lowest $N$ levels of a set of suitably defined orthonormal orbitals. The set-indices in the fixed- $N$ Hilbert space, $J \rightarrow\left\{p_{1}, \cdots, p_{m} ; \alpha_{1}, \cdots, \alpha_{m}\right\}$ now represent an $m$-particle/ $m$-hole cluster configuration, where the single-particle indices $\left\{\alpha_{i}\right\}$ label the single-hole states that can be created by removing an electron from $|\Phi\rangle$, and the single-particle indices $\left\{p_{i}\right\}$ label the remaining single-particle states unoccupied in $|\Phi\rangle$.

The CCM is now characterized by writing the exact ground ket state $\left|\Psi_{0}\right\rangle$ of the many-body Hamiltonian $H$ in terms of an exponentiated cluster correlation operator $S$,

$$
\left|\Psi_{0}\right\rangle=\mathrm{e}^{S}|\Phi\rangle ; \quad H\left|\Psi_{0}\right\rangle=E_{0}\left|\Psi_{0}\right\rangle,
$$

which may be exactly decomposed in terms of creation operators only,

$$
S=\sum_{J}^{\prime} \mathcal{S}_{J} C_{J}^{\dagger}
$$

where, by convention, a prime on the sum over configurations $\{J\}$ excludes the term $J=0$ corresponding to the identity operator, $C_{0}^{\dagger} \equiv I$. We thus have the intermediate normalization condition fulfilled, namely $\left\langle\Phi \mid \Psi_{0}\right\rangle=\langle\Phi \mid \Phi\rangle=1$, in the usual scheme where $\left\langle\Phi\left|C_{J}^{\dagger}\right| \Phi\right\rangle=0, \forall J \neq 0$. The cluster correlation operator of an $N$-body system may also be decomposed as a linear superposition of $n$-body partitions, $S=\sum_{n=1}^{N} S_{n}$, where $S_{n}$ describes those configurations formed by exciting $n$ particles from their singleparticle states occupied in $|\Phi\rangle$.

The reason for the exponentiated form of the correlation operator in Eqs. (62) and (63), rather than the linear form of Eq. (59) which characterizes the configurationinteraction (CI) method, lies at the heart of the CCM. ${ }^{6,11,13,96}$ Particularly important features of the exponential form are: (i) its proper counting of independent excitations (e.g., $n$ independent pairs of particles excited from $|\Phi\rangle$ are properly described by the wave function $\left.\frac{1}{n !} S_{2}^{n}|\Phi\rangle\right)$; (ii) its automatic incorporation of the important linked-cluster theorem of Goldstone; and (iii) its incorporation of size-extensivity, so that extensive variables such as the ground-state energy $E_{0}$ scale properly with particle number in the infinite limit, $N \rightarrow \infty$, even when $S$ is truncated.

In practice the CCM is implemented by first restricting the cluster amplitudes $\left\{\mathcal{S}_{J}\right\}$ to some finite or infinite subset of the complete set, within some well-defined hierarchy of such approximations. Solutions for the retained amplitudes are then found by first re-writing the ground-state Schrödinger equation (62) in the similarity-transformed form,

$$
\left(\mathrm{e}^{-S} H \mathrm{e}^{S}-E_{0}\right)|\Phi\rangle=0
$$

which is characteristic of the CCM. By taking the inner products of Eq. (64) with the complete set of states comprising $|\Phi\rangle$ itself and $\left\{C_{J}^{\dagger}|\Phi\rangle ; J \neq 0\right\}$, we find respectively,

$$
\left\langle\Phi\left|\mathrm{e}^{-s} H \mathrm{e}^{S}\right| \Phi\right\rangle=E_{0}
$$




$$
\left\langle\Phi\left|C_{J} \mathrm{e}^{-S} H \mathrm{e}^{S}\right| \Phi\right\rangle=0 ; \quad J \neq 0 .
$$

The set of equations (66) represents a coupled set of nonlinear equations for the $c$ number coefficients $\left\{\mathcal{S}_{J}\right\}$, equal in number to the number of retained configurations $\{J\}$. In terms of their solutions, Eq. (65) then gives the corresponding approximant to the ground-state energy, $E_{0}=E_{0}\left[\mathcal{S}_{J}\right]$.

The well-known nested commutator expansion,

$$
\mathrm{e}^{-S} H \mathrm{e}^{S}=H+[H, S]+\frac{1}{2 !}[[H, S], S]+\cdots,
$$

together with the mutual commutativity of each of the individual terms in the expansion of Eq. (63), ensures that every element of $S$ in Eq. (63) is linked directly to the Hamiltonian in each of the terms in Eq. (67). No unlinked (or, equivalently, disconnected) terms or diagrams can arise, and each of the coupled equations (66) is of linked-cluster type. Also, when each term in the Hamiltonian $H$, written in secondquantized form, contains only a finite number of single-particle destruction operators defined with respect to $|\Phi\rangle$, as is usually the case, the otherwise infinite series of Eq. (67) terminates after a finite number of terms. As a result the left-hand sides of Eqs. $(65)$ and $(66)$ are of finite order in the retained coefficients $\left\{\mathcal{S}_{J}\right\}$, and no further (artificial or approximate) truncation needs to be made for their evaluation. The CCM thus differs fundamentally from its unitarity-transformation counterpart that would arise in a standard variational treatment, in which the bra state $\left\langle\Psi_{0}\right|$ is simply taken as the explicit Hermitian conjugate $\langle\Phi| e^{S^{\dagger}}$ of $\left|\Psi_{0}\right\rangle$.

The above feature is very important, and it leads naturally to the biorthogonal description of states within the CCM (rather than the more usual orthogonal description), in which the manifest Hermitian conjugacy of corresponding bra and ket states is not imposed from the outset. While the bra states, as we have seen above, are not required for the evaluation of the ground-state (g.s.) energy, they are needed to calculate other g.s. expectation values, such as order parameters. In what has become known ${ }^{96}$ as the normal version of the CCM (or NCCM), the bra ground state is parametrized in the form,

$$
\begin{gathered}
\left\langle\tilde{\Psi}_{0}\right|=\langle\Phi| \tilde{S}^{-S} ; \quad\left\langle\tilde{\Psi}_{0}\right| H=E_{0}\left\langle\tilde{\Psi}_{0}\right|, \\
\tilde{S}=1+\sum_{J}^{\prime} \tilde{\mathcal{S}}_{J} C_{J},
\end{gathered}
$$

with the explicit normalization,

$$
\left\langle\tilde{\Psi}_{0} \mid \Psi_{0}\right\rangle=\left\langle\Phi \mid \Psi_{0}\right\rangle=\langle\Phi \mid \Phi\rangle=1
$$

Equation (68) readily leads to the equations,

$$
\left\langle\Phi\left|\tilde{S}\left(\mathrm{e}^{-S} H \mathrm{e}^{S}-E_{0}\right) C_{J}^{\dagger}\right| \Phi\right\rangle=0, \quad \forall J,
$$

which, by making use of Eq. (65) and the fact that $\left[S, C_{J}^{\dagger}\right]=0, \forall J$, may easily be rewritten as,

$$
\left\langle\Phi\left|\tilde{S} \mathrm{e}^{-S}\left[H, C_{J}^{\dagger}\right] \mathrm{e}^{S}\right| \Phi\right\rangle=0, \quad \forall J .
$$

Equations (71) and (72) have the form of coupled but linear equations for the brastate coefficients $\left\{\tilde{\mathcal{S}}_{J}\right\}$ once the ket-state coefficients $\left\{\mathcal{S}_{J}\right\}$ are used as known input. The resulting equations again contain only finite powers of $S$ since the nested commutator expansions terminate as before. One may readily show that Eq. (71) has the formal solution,

$$
\langle\Phi| \tilde{S}=\langle\Phi|-\langle\Phi| \mathrm{e}^{-S} H \mathrm{e}^{S} Q\left(Q \mathrm{e}^{-S} H \mathrm{e}^{S} Q-E_{0}\right)^{-1} Q,
$$




$$
Q \equiv I-|\Phi\rangle\langle\Phi|
$$

in terms of which the g.s. expectation value of an arbitrary operator $A$ can be written as,

$$
\begin{aligned}
\bar{A} & =\bar{A}\left[\mathcal{S}_{J}\right] \equiv\left\langle\tilde{\Psi}_{0}|A| \Psi_{0}\right. \\
& =\left\langle\Phi\left|\mathrm{e}^{-S} A \mathrm{e}^{S}\right| \Phi\right\rangle-\left\langle\Phi\left|\mathrm{e}^{-S} H \mathrm{e}^{S}\left(Q \mathrm{e}^{-S} H \mathrm{e}^{S} Q-E_{0}\right)^{-1} Q \mathrm{e}^{-S} A \mathrm{e}^{S}\right| \Phi\right\rangle .
\end{aligned}
$$

We note that Eq. (66) guarantees that $\tilde{H}=E_{0}$, as given by Eq. (65).

Excited states $\left\{\left|\Psi_{\lambda}\right\rangle\right\}$ are described within the $\mathrm{CCM}^{97}$ by linear excitation operators $\left\{X^{\lambda}\right\}$ which act on the corresponding exact g.s. wave function $\left|\Psi_{0}\right\rangle$,

$$
\left|\Psi_{\lambda}\right\rangle=X^{\lambda}\left|\Psi_{0}\right\rangle=X^{\lambda} \mathrm{e}^{S}|\Phi\rangle ; \quad H\left|\Psi_{\lambda}\right\rangle=E_{\lambda}\left|\Psi_{\lambda}\right\rangle \equiv\left(E_{0}+\epsilon_{\lambda}\right)\left|\Psi_{\lambda}\right\rangle,
$$

where $X^{\lambda}$ is again decomposable wholly in terms of creation operators defined with respect to $|\Phi\rangle$,

$$
X^{\lambda}=\sum_{J}^{\prime} \mathcal{X}_{J}^{\lambda} C_{J}^{\dagger}
$$

Hence, the operators $S$ and $X^{\lambda}$ commute. The excited-state Schrödinger equation (76) may be combined with its g.s. counterpart of Eq. (62) to give the CCM eigenvalue equation,

$$
\mathrm{e}^{-S}\left[H, X^{\lambda}\right] \mathrm{e}^{S}|\Phi\rangle=\epsilon_{\lambda} X^{\lambda}|\Phi\rangle
$$

for the excitation energy, $\epsilon_{\lambda} \equiv E_{\lambda}-E_{0}$, directly.

Equation (78) may be equivalently written as,

$$
\left(Q \mathrm{e}^{-S} H \mathrm{e}^{S} Q-E_{0}\right) Q X^{\lambda}|\Phi\rangle=\epsilon_{\lambda} Q X^{\lambda}|\Phi\rangle,
$$

from which we see that the excitation spectrum is found by diagonalizing, in the space spanned by the retained states $\left\{C_{J}^{\dagger}|\Phi\rangle ; J \neq 0\right\}$, the same matrix $\left(Q \mathrm{e}^{-S} H \mathrm{e}^{S} Q-E_{0}\right)$ as needs to be inverted in Eq. (73) to find $\tilde{S}$. In all practical applications of the CCM this space is truncated to some (finite or infinite) subspace spanned by a subset of the states. The operators $S, \tilde{S}$, and $X^{\lambda}$ given by Eqs. (63), (69), and (77) are thus approximated by restricting the sum over the configurations $\{J\}$ to the appropriate subset.

\section{Features of the CBF and CCM Techniques}

The CBF method and the CCM have a number of highly desirable features. We list some of the more important of these below.

- Both methods are extremely versatile, and they have been extensively tested. There is by now a large amount of experience in handling them.

- An impressively wide range of applications to systems of physical interest has been made of one and/or the other method. These include finite nuclei; nuclear matter; quantum field theory (including systems of anharmonic oscillators, $\phi^{4}$ field theory, and pion-nucleon field theory); atoms and molecules of interest in quantum chemistry; the electron gas; quantum hydrodynamics; and the liquids helium (including bulk ${ }^{3} \mathrm{He}$ and ${ }^{4} \mathrm{He}$ and their mixtures, and films).

- Both methods are capable of very high accuracy at attainable levels of implementation. In most applications the CBF and/or CCM results are either the best or among the best from all available microscopic techniques. They are now often at the point of being competitive with the large-scale Monte Carlo simulations in the cases where the latter can be performed. 
- Neither method is restricted in principle to particular forms of the Hamiltonian. Both are easily capable of handling very complicated interactions.

- Both the CBF and CCM are intrinsically nonperturbative in nature. Some correlations are retained to infinite order, even at the lowest levels of implementation. The CCM, in particular, can often be used to derive (or reconstruct) perturbation theory (PT) series, by a suitable choice of truncation hierarchy for the subsets of terms retained in the multiconfigurational expansions of the correlation operators $S, \tilde{S}$, and $X^{\lambda}$. In such cases the CCM provides a natural analytic continuation of the PT series, which in practice is usually found to be valid far outside the radius of convergence of the PT series, and also to be quantitatively superior to such alternative schemes as (generalized) Padé resummations.

- Although nonperturbative in principle, the CCM can be easily related to the Goldstone diagram expansions of time-independent perturbation theory. This feature facilitates comparisons with other methods.

- Similarly, at the optimized Jastrow level implemented via the hypernetted chain approximation, the CBF method has been shown ${ }^{98}$ to be equivalent to two-body localized parquet theory, and hence to a sum of planar Feynman diagrams of timedependent perturbation theory.

- In both methods one may work from the outset in the bulk limit, $N \rightarrow \infty$, thereby avoiding problems connected with finite-size effects.

- Both methods have the virtue of great flexibility. One can choose "uncorrelated" or "model" g.s. reference states $\left|\Phi_{0}\right\rangle$, for example, in many ways. In particular, this presents an opportunity for the 'conceptual' school of many-body theory to provide a good starting-point for the 'quantitative' school. Similarly, many different approximation hierarchies for the correlation operators $F$ and $S$ of the CBF and CCM schemes respectively can be envisaged, and there is again room for outside experience or physical intuition to be utilized in their choice.

- Both methods are capable of handling phase transitions, both in theory and in practice. Even when $\left|\Phi_{0}\right\rangle$ is a poor choice, both the CBF and CCM schemes have been shown in particular cases to be able to predict phase changes.

- Both methods, but particularly the CCM, often have the practical capability of implementation at high orders of approximation. The CCM has especially been shown to be very amenable to the use of computer algebra to derive the high-order basic coupled sets of nonlinear equations (66) which underpin it. This feature is particularly marked for lattice systems, and it is a key reason why the CCM is now proving to be fully competitive with large-scale stochastic simulations at a fraction of the computing cost, in those cases where the latter can be performed.

These combined features make both the CBF and CCM schemes extemely attractive candidates for providing a unified treatment of lattice Hamiltonian systems of the sort described in the three preceeding Sections. In the following Section we now give a brief overview of the applications that have been made to date. 


\section{TYPICAL CBF AND CCM RESULTS}

\section{Spin-Lattice Systems}

Variational wave functions of the Jastrow form have been studied by several authors $^{9-103}$ in the context of such spin-lattice problems as the spin- $\frac{1}{2} X X Z$ model on a bipartite lattice. We now start from a model state $\left|\Phi_{0}\right\rangle$ which is just the twosublattice perfectly aligned spin- $\frac{1}{2}$ Néel state $|N 2\rangle$ in which all spins on one sublattice are aligned along the $+z$-axis, and all those on the other sublattice along the $-z$-axis. The ground-state (g.s.) Jastrow wave function can then be written as,

$$
\left|\Psi_{0}^{J}\right\rangle=\mathcal{N} \exp \left(-\frac{1}{2} \sum_{i<j} u_{i j} \sigma_{i}^{x} \sigma_{j}^{x}\right)|\mathrm{N} 2\rangle
$$

where $\mathcal{N}$ is a normalization constant, and the mode of action of the operator $\sigma_{k}^{x}$ on the spin- $\frac{1}{2}$ Néel state is easily seen to be to flip the direction of the spin (from up to down, or vice versa) on the lattice site $k$. Recent calculations of this sort ${ }^{102}$ have used the form of trial wave function given in Eq. (80) in the variational Monte Carlo approach.

Most interestingly for present purposes, Manousakis ${ }^{104}$ has also performed for the bipartite spin- $\frac{1}{2}$ Heisenberg model a paired magnon analysis, which is the precise counterpart of the paired phonon analysis (PPA) for a continuous bosonic system in usual CBF theory. ${ }^{16,17}$ In particular, he defines a complete and orthonormal set of multimagnon states, and calculates the matrix elements of the Hamiltonian using a separability approximation discussed by Feenberg ${ }^{16}$ for the PPA. In this way he finds a wave function of precisely the Jastrow form of Eq. (80) with a specific form of the spatial correlation function, $u_{i j}=u\left(\mathbf{R}_{i}-\mathbf{R}_{j}\right)$, where $\mathbf{R}_{k}$ is the position vector of lattice site $k$, which exactly reproduces the results (for the ground-state energy, single-magnon excitation energy dispersion relation, and staggered magnetization, for example) of linear spin-wave theory (SWT) ${ }^{105}$ first introduced by Anderson.

The above interesting result of Manousakis ${ }^{104}$ suggests a way to improve systematically upon linear SWT as an alternative to the more usual nonlinear versions of SWT which involve typically a perturbation series in powers of $(1 / s)$, where $s$ is the spin quantum number; a loop expansion in powers of $(1 / z)$, where $z$ is the coordination number of the lattice (i.e., the number of nearest neighbours to a given site); or other modified versions. ${ }^{106,107}$ Thus, one could now imagine performing any of the higher-order versions of CBF theory discussed in the preceeding Section to go beyond the paired magnon approximation or, effectively, beyond linear SWT. An obvious first such attempt might be to perform CBF perturbation theory in a basis of correlated multimagnon states generated by letting the Jastrow correlation operator, $F_{J} \equiv \exp \left(-\frac{1}{2} \sum_{i<j} u_{i j} \sigma_{i}^{x} \sigma_{j}^{x}\right)$, act as in $\mathrm{Eq} .(50)$ on a set of reference states. To the best of our knowledge, no such higher-order CBF calculations have actually yet been performed for spin-lattice problems, although it would clearly be most interesting to explore this field further.

By contrast, the CCM has begun to be applied quite intensively over the last few years. ${ }^{108-116}$ For example, applications have by now been made to the solid phases of ${ }^{3} \mathrm{He}^{108}$ the isotropic (Heisenberg) and anisotropic $X X Z$ models in $1 \mathrm{D}$ and on the $2 \mathrm{D}$ square lattice, both for spin- $\frac{1}{2}$ systems $^{108-110,113,115,116}$ and higher-spin systems; ${ }^{111}$ the spin- $\frac{1}{2} J_{1}-J_{2}$ or Majumdar-Ghosh (MG) model of Eq. (21), for the $1 \mathrm{D}$ chain and 2D square lattice; ${ }^{114}$ and the spin-1 Heisenberg-biquadratic (HB) model of Eq. (20) in 1D. ${ }^{112}$ We give a very brief review of these results below.

In order to perform a CCM analysis one must first choose a model state $|\Phi\rangle$, as indicated in the preceeding Section. Although there are many alternative choices, ${ }^{112,114(b), 115}$ 
we illustrate the approach here with what is probably the simplest and most obvious choice for bipartite-lattice antiferromagnetic systems, namely the (classical) Néel state $|\mathrm{N} 2\rangle$, in which all spins on the $A$-sublattice have $z$-component $s^{z}=-s$, say, and all those on the $B$-sublattice have $s^{z}=+s$. The single-spin creation operators with respect to $|\mathrm{N} 2\rangle$ are clearly the spin-raising operators $\left\{s_{i}^{+}\right\}$for sites $i$ on the $A$-sublattice and the spin-lowering operators $\left\{s_{j}^{-}\right\}$for sites $j$ on the $B$-sublattice, where $s_{k}^{ \pm} \equiv s_{k}^{x} \pm i s_{k}^{y}$. Each of these operators can act a maximum of $2 s$ times on a single site before the state $|\mathrm{N} 2\rangle$ is annihilated.

When the Hamiltonian commutes with $s_{T}^{z}$, as in Eqs. (5) and (6) for the $X X Z$ model, the antiferromagnetic ground state $\left|\Psi_{0}\right\rangle$ may be sought in the sector where $s_{T}^{z}=0$. Equations (62) and (63) thus become, in this case,

$$
\left|\Psi_{0}\right\rangle=\mathrm{e}^{S}|\mathrm{~N} 2\rangle ; \quad S=\sum_{m=1}^{N / 2} S_{2 m}
$$

where $N \rightarrow \infty$ is the total number of spins, and with cluster correlation operators $S_{2 m}$ expressed with a somewhat arbitrary choice of normalization constant as,

$$
S_{2 m}=\frac{(-1)^{m}}{(m !)^{2}} \sum_{i_{1}, i_{2}, \cdots i_{m}} \sum_{j_{1}, j_{2}, \cdots j_{m}} \mathcal{S}_{i_{1} i_{2} \cdots i_{m} ; j_{1} j_{2} \cdots j_{m}} s_{i_{1}}^{+} s_{i_{2}}^{+} \cdots s_{i_{m}}^{+} s_{j_{1}}^{-} s_{j_{2}}^{-} \cdots s_{j_{m}}^{-},
$$

with equivalent analogues of Eqs. (68) and (69). Clearly, for spin-s particles, the coefficients $\mathcal{S}_{i_{1} \cdots i_{m} ; j_{1} \cdots j_{m}}$ vanish if any one of the lattice indices appears more than $2 s$ times.

The ground-state energy $E_{0}$ and the coupled set of equations for the coefficients $\left\{\mathcal{S}_{i_{1} \cdots i_{m} ; j_{1} \cdots j_{m}}\right\}$ are now obtained from the general Eqs. (65)-(67). The algebraic form of the latter is that of a coupled set of multinomial equations. For the $X X Z$ Hamiltonian of Eq. (4), for example, these are easily seen to be of degree no higher than fourth, by noting the bilinear form of the Hamiltonian and the specific $S U(2)$ commutation relations to be used in Eq. (67). Their explicit evaluation is straightforward, although very tedious and time-consuming if done by hand if more than a few configuration coefficients are retained in the expansion of the correlation operator $S$ from Eqs. (81) and (82). Nevertheless, the algebraic reduction is very amenable to automation by the use of computer algebra, and this makes the accurate implementation of the CCM to high orders very tractable and attractive in practice.

All that remains to implement the CCM is to invoke a suitable truncation scheme for the cluster operator $S$. These cannot ever be uniquely or universally specified, but need to be motivated on physical grounds for the problem at hand. We discuss here three examples of truncation methods that have been applied to spin-lattice problems. They are by no means the only possible choices. Firstly, we have the so-called SUB $n$ scheme in which the summation in Eq. (81) is truncated at the upper limit $m=\frac{1}{2} n$, so that one retains only correlations in $S$ involving $n$ or fewer spins. Secondly, one may consider the simpler SUBn-m sub-approximation to the $\mathrm{SUB} n$ scheme, where only those $\mathrm{SUB} n$ correlations spanning a range of no more than $m$ adjacent lattice sites are included. Thirdly, we have also studied the systematic local LSUBm scheme, which includes all possible multi-spin correlations over a specified locale on the lattice, where $m$ is the nominal index that characterizes the size of the given locale. Thus, for the specific problem of a spin- $\frac{1}{2}$ system on a bipartite lattice, the LSUB $m$ scheme retains only those configurations in $S$ which contain any number of flipped spins with respect to the Néel model state over a localized region of at most $m$ contiguous sites on the lattice, and which are compatible with the condition that $s_{T}^{z}=0$. In all of the above cases the remaining correlation coefficients in $S$ are set to zero. 


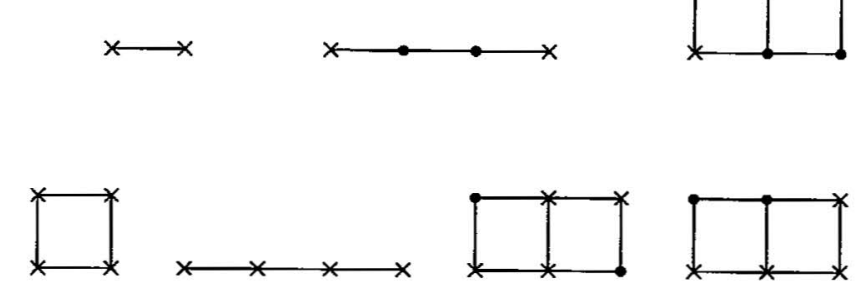

Figure 1. The seven independent configurations retained in the LSUB4 scheme for spin- $\frac{1}{2}$ systems on the $2 \mathrm{D}$ square lattice. The three configurations in the first row represent the corresponding SUB2-4 coefficients. The crosses indicate the positions of the spins which are flipped with respect to the Néel model state.

We first describe the CCM results obtained for the spin- $\frac{1}{2} X X Z$ models employing the above truncations with respect to the Néel model state. To date, the most extensive $\mathrm{LSUB} m$ results have been given ${ }^{116}$ for $m \leq 10$ for the $1 \mathrm{D}$ chain and for $m \leq 6$ for the $2 \mathrm{D}$ square lattice. It should be noted that all of these calculations were done on microcomputers. The 1D LSUB10 calculation involves 81 independent spin-flip configurations. The corresponding numbers in the LSUB4 and LSUB6 calculation on the square lattice are 7 and 72 . We illustrate the former of these in Fig. 1.

For the $1 \mathrm{D}$ chain it has been demonstrated explicitly ${ }^{110}$ that for a given value of $m$ the LSUB $m$ scheme reproduces exactly the corresponding $2 m$ th order of large- $\Delta$ perturbation theory, and that the LSUB $m$ scheme also gives good results in the planar region $(|\Delta|<1)$ where perturbation theory is not valid. At the critical isotropic point $(\Delta=1)$ the LSUB10 approximation yields a value -0.4420 for the ground-state (g.s.) energy per spin, $E_{0} / N$. Naive attempts to extrapolate the LSUB $m$ results to the exact $m \rightarrow \infty$ limit indicate that a $1 / \mathrm{m}^{2}$ rule fits the numerical values well. The g.s. energy per spin after this extrapolation, using the calculated values with $m=4,6,8,10$, yields $-0.4431 \pm 0.0001$, while the exact number by the Bethe ansatz solution from Eqs. (8) or (9) is -0.4432 to the accuracy of four significant figures.

In Fig. 2 we show comparable LSUB $m$ results for the g.s. energy of the spin- $\frac{1}{2} 2 \mathrm{D}$ square lattice, ${ }^{116}$ together with those from a Monte Carlo calculation ${ }^{117}$ on an $8 \times 8$ lattice for comparison. One observers the excellent agreement over a wide range of values of the anisotropy parameter $\Delta$. At the isotropic point $(\Delta=1)$, for example, the LSUB6 result for the g.s. energy per spin is -0.6670 . The extrapolated "LSUB $\infty$ " result, using the same $1 / \mathrm{m}^{2}$ rule as for the $1 \mathrm{D}$ case, is $-0.6691 \pm 0.0003$. This value compares very well with the most accurate values available of $-0.66934 \pm 0.00003$ from a series of large-scale Monte Carlo simulations by Runge ${ }^{44}$ on lattices up to size $16 \times 16$, and $-0.6694 \pm 0.0001$ from the series expansion technique. ${ }^{118}$ It is interesting to note that in terms of relative error, the LSUB6 result by comparison with the exact infinitelattice result is comparable in accuracy to that of a $10 \times 10$ finite lattice, for which the Monte Carlo estimate is -0.6715 .

A particularly interesting feature of the above LSUB $m$ results is the appearance of terminating points in the solutions for $m>2$ for the $2 \mathrm{D}$ square lattice, as indicated in Fig. 2. By contrast, no such behaviour is observed in $1 \mathrm{D}$ at any value $m \leq 10 .{ }^{110}$ The numerical results for the anisotropic susceptibility, $\chi_{a} \equiv-\partial^{2}\left(E_{0} / N\right) / \partial \Delta^{2}$, as a function of $\Delta$ are shown in Fig. 3 for both cases. It is clear that there is no singular behaviour for the $1 D$ chain. This is in accord with the exact expressions of Eqs. (8) and (9) from 


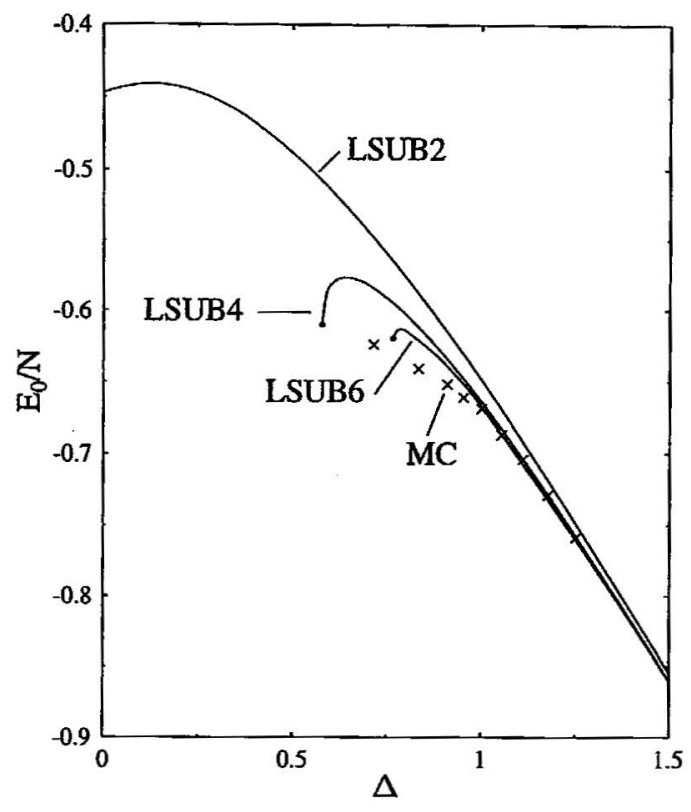

Figure 2. Ground-state energy per spin as a function of $\Delta$ for the spin- $\frac{1}{2} X X Z$ model on the 2D square lattice. Shown are the results of the CCM in the LSUBm scheme with $m=2,4,6$ from Ref. 116 , and of the Monte Carlo (MC) calculation of Ref. 117.

the Bethe ansatz, which give an essential singularity at $\Delta=1$, with the result that any finite order of derivative with respect to $\Delta$ is continuous. By contrast, the anisotropic susceptibility for the $2 \mathrm{D}$ square lattice model clearly shows a singular behaviour. It is shown in Ref. 116 how the CCM analysis allows a detailed study of the quantum critical behaviour in a systematic and unbiased manner, such that predictions for both the critical value $\Delta_{c}$ of the phase transition and the corresponding critical exponents can be made.

Other quantities like the staggered mangetization and spin-spin correlation function can also be calculated. For example, at the isotropic point $(\Delta=1)$ for the $2 \mathrm{D}$ square lattice, the LSUB $m$ approximations for $M^{z}$ are $0.8514,0.7648$, and 0.7278 for $m=2,4$, and 6 , respectively, with an extrapolated "LSUB $\infty$ " estimate of $0.68 \pm 0.01$. This prediction that the 2D Heisenberg model on the square lattice has nonzero Néel order, unlike the $1 \mathrm{D}$ chain, is in accord with other calculations. For example, the corresponding values for $M^{z}$ from linear spin-wave theory ${ }^{105}$ and series expansion techniques ${ }^{118}$ are 0.606 and $0.62 \pm 0.02$, respectively, whereas the best of the Monte Carlo results ${ }^{44}$ vary between $0.68 \pm 0.02$ and $0.62 \pm 0.04$. The interested reader is referred to the literature ${ }^{110,116}$ for further details of the CCM results for this model in LSUBm approximations.

Compared to the LSUBm sequence of approximations, which retain only a finite number of localized multi-spin-flip configurations with respect to the model Néel state for any finite value of $m$, even the lowest level $\mathrm{SUB} n$ approximations retain an infinite set of spin-flip configurations, some of which are of arbitrarily long range. Thus, for 


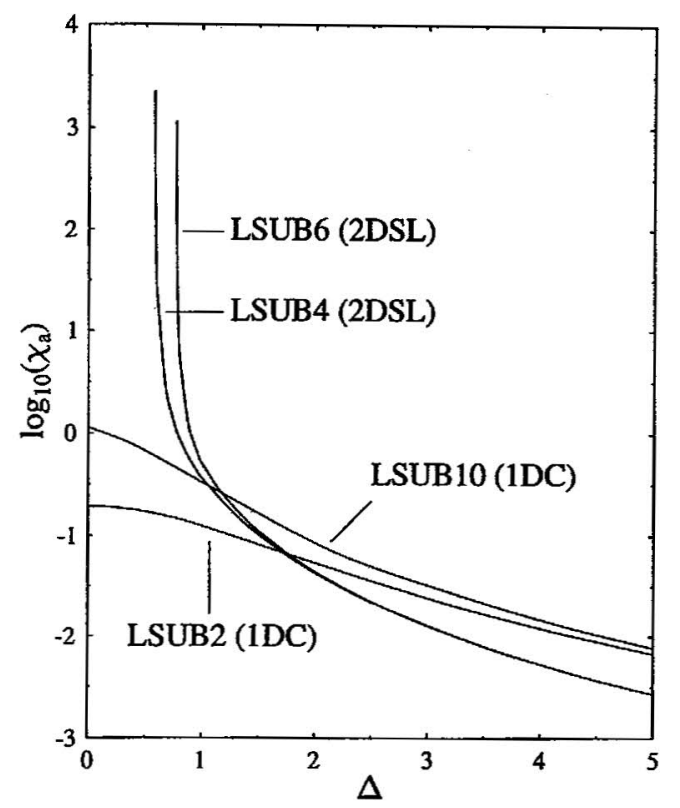

Figure 3. The second-order derivative of the ground-state energy per spin with respect to $\Delta$ for the spin- $\frac{1}{2} X X Z$ model on a $1 \mathrm{D}$ chain (1DC) and the 2D square lattice (2DSL). The results are from a CCM calculation of Ref. 116 in various LSUBm schemes.

example, the SUB2 wave function,

$$
\left|\Psi_{0}^{\mathrm{SUB} 2}\right\rangle=\exp \left(-\sum_{i \in A} \sum_{j \in B} \mathcal{S}_{i j} s_{i}^{+} s_{j}^{-}\right)|\mathrm{N} 2\rangle,
$$

where $\mathcal{S}_{i j}=\mathcal{S}\left(\mathbf{R}_{i}-\mathbf{R}_{j}\right)$, with $\mathbf{R}_{k}$ the position vector of lattice site $k$, retains all 2spin-flip configurations. It is not, therefore, perhaps too surprising that even the lowest level $\mathrm{SUB} n$ approximations can predict phase transitions.

Indeed, the CCM SUB2 approximation based on the Néel model state has been applied, for example, to the $X X Z$ model for arbitrary values of the spin quantum number $s,{ }^{111}$ where it yields a prediction of the critical value $\Delta_{c}$ of the anisotropy parameter for each $s$ for the Heisenberg-Ising phase transition. It is found that the CCM solution for $\mathcal{S}_{i j}$ exists in each case only for $\Delta>\Delta_{c}=\Delta_{c}(s)$. Precisely at the critical point both the staggered magnetization and the gap in the excitation spectrum are shown to be precisely zero when calculated at the same level of approximation, ${ }^{110,111}$ just as in the case of the transition which is known to be exactly at $\Delta=1$ for the spin $-\frac{1}{2}$ case in $1 \mathrm{D}$. The value of $\Delta_{c}^{\mathrm{SUB2}}$ is also found to approach the classical value of unity as the spin quantum number $s$ increases, as can be seen from Fig. 4, which illustrates nicely the approach to the classical limit, $s \rightarrow \infty$, at this level of approximation.

We note the similarities as well as the important differences between the CCM form of the wave function in SUB2 approximation, given by Eq. (83), with the paired magnon and Jastrow form of Eq. (80). The linear SWT wave function can also be written in precisely the same form as that in Eq. (83), ${ }^{103}$ and the relationship between the CCM SUB2 and linear SWT approximations and their corresponding wave functions has been investigated in some detail in Ref. 111. It would now be interesting, especially in view of the paired magnon analysis of Manousakis ${ }^{104}$ discussed above, also to investigate the 


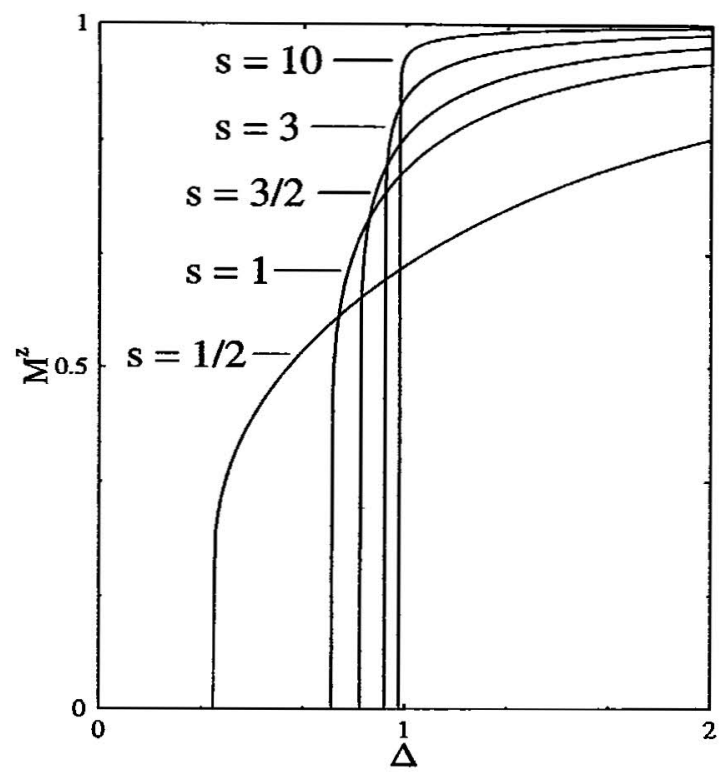

Figure 4. Staggered magnetization as a function of $\Delta$ for the 1D $X X Z$ model for various values of the spin quantum number $s$. The results are obtained in the SUB2 CCM approximation based on a Néel model state, and are taken from Ref. 111.

detailed relationship between the linear SWT and CBF paired magnon analysis wave functions.

Finally, we note that the CCM analyses and approximation schemes discussed above can be repeated for different choices of model reference states $|\Phi\rangle$, perhaps more appropriate to other systems or other specific phases under consideration. Such calculations have been performed, for example, for (i) the spin- $\frac{1}{2}$ Majumdar-Ghosh model, using both the Néel model state ${ }^{114(a)}$ and a dimerized model state; ${ }^{114(b)}$ (ii) the spin1 Heisenberg-biquadratic $1 \mathrm{D}$ chain, ${ }^{112}$ using both the Néel model state and a planar model state $|N 1\rangle$ defined to have $s_{k}^{z}=0$ on all lattice sites $k$; and (iii) the spin- $\frac{1}{2} X X Z$ model on the 1D chain, where the model is first exactly fermionized using the JordanWigner transformation (so that the $X Y$ model obtained at $\Delta=0$ is mapped onto a free fermionic model), and the model state is then taken to be the filled Fermi sea wave function of the free fermions. ${ }^{115}$ This latter calculation, for example, thus provides a means of tailoring the model state to deal with the planar phase in the regime $|\Delta|<1$, by contrast with the previous Néel state choice which is more suited to the Ising-like phase for $\Delta>1$.

The interested reader is referred to the cited literature for further details of all these calculations and, in particular, how suitable sets of (composite) creation and destruction operators can be defined with respect to the above alternative model states as cyclic vectors, as required by the CCM approach.

\section{Electron-Lattice Systems}

After the above discussion it is natural to turn next to the problem of a single vacancy in a quantum antiferromagnet, and to consider the resulting hole motion in, 
for example, a state with nonvanishing Néel order, via the $t$ - $J$ model. The important physical effects that need to be included in principle are: (i) the quantum fluctuation effects which excite and de-excite spin waves; (ii) renormalization effects due to the emission and absorption of spin waves; and (iii) the distortion of the spin background due to the vacancy, which is the spin polaron problem. Not all microscopic calculations are designed to describe all of these effects.

There have been several variational approaches to the problem of a single vacancy on a quantum antiferromagnet, ${ }^{103,119}$ of which the calculation of Sachdev ${ }^{103}$ is typical. After considering the form of the wave function obtained in linear SWT for the Nélordered ground state of a spin- $\frac{1}{2}$ Heisenberg antiferromagnet on a $2 \mathrm{D}$ square lattice, he used a similar form as a trial variational wave function. He then further generalized it to the case where a single hole is present. This form of the wave function essentially neglects the renormalization effect (ii) above.

For higher concentrations of vacancies variational calculations have also been performed for both the $t-J$ and Hubbard models. ${ }^{120}$ The CBF method has also been employed for the Hubbard model ${ }^{121,122}$ using a lattice version of the Fermi hypernetted chain (FHNC) technique. ${ }^{90}$ These very interesting CBF calculations have been performed for a variety of both reference states $\left|\Phi_{0}\right\rangle$ and correlation operators $F$ in Eq. (50). In the first place, a number of different correlation effects were incorporated on top of the paramagnetic filled Fermi sea state $|F\rangle$ (or Slater determinant of singleparticle plane waves) as model state $\left|\Phi_{0}\right\rangle$. Those correlations considered included the following:

- a Gutzwiller on-site correlation generated by the state-independent operator of Jastrow form,

$$
F_{G}=\prod_{i<j}\left[1-(1-g) \delta_{i \uparrow, j \downarrow}\right] ;
$$

- a more general state-independent Jastrow correlation operator of the form of Eq. (52), which also includes nearest-neighbour correlations, namely where

$$
f\left(r_{i j}\right) \longrightarrow \begin{cases}g, & r_{i j}=0 \\ g_{1}, & r_{i j}=\rho \\ 1, & \text { otherwise }\end{cases}
$$

where $\rho$ is a nearest-neighbour vector on the lattice;

- a so-called quartet correlation operator, which is designed to mimic the excitonic (bound-state) correlations between empty and doubly-occupied sites discussed by earlier authors; ${ }^{123}$ and

- a spin-spin correlation operator akin to that of Eq. (54), which correlates nearestneighbour spins.

Since the Fermi liquid versus Luttinger liquid nature of the $2 \mathrm{D}$ Hubbard model is of great interest, the same authors ${ }^{121,122}$ have also performed similar CBF investigations based on two examples of model wave functions $\left|\Phi_{0}\right\rangle$ without a sharp Fermi surface. In the first place, it was shown some time ago ${ }^{124}$ how a BCS wave function could be employed as the starting point for a CBF-FHNC analysis, and a preliminary analysis of the Gutzwiller-correlated BCS state for the 1D Hubbard model has been presented. ${ }^{121}$ Perhaps more interestingly, an (uncorrelated) model spin-density wave state, $|S D W\rangle$, which has antiferromagnetic long-range order, has also been employed in a CBF analysis of the $1 \mathrm{D}$ Hubbard chain at half filling. ${ }^{122}$ 
The correlated spin-density wave state is a band antiferromagnetic ground state with spatial periodicity $\pi / k_{F}$, and the model state $|\mathrm{SDW}\rangle$ is just a magnetic band insulator at any filling. The Fermi surface is trivially destroyed by single-particle magnetic Bragg scattering. The model state $|\mathrm{SDW}\rangle$ is a simple Slater determinant formed from singleparticle wave functions which are a product of a spatial function,

$$
\phi_{\mathbf{k} \sigma}\left(\mathbf{r}_{i}\right)=u_{k} \mathrm{e}^{i \mathbf{k} \cdot \mathbf{r}_{i}}+\operatorname{sgn}(\sigma) v_{k} \mathrm{e}^{i(\mathbf{k}+\mathbf{Q}) \cdot \mathbf{r}_{i}} ; \quad k \leq k_{F}, \quad u_{k}^{2}+v_{k}^{2}=1
$$

where $\operatorname{sgn}(\sigma)= \pm 1$ according as the spin projection $\sigma=2 s^{z}= \pm 1$, with a spin wave function. The vector $\mathbf{Q}$ in Eq. (86) is simply the wave vector characterizing the extra periodicity of the antiferromagnetic ordering. It could in principle itself be determined variationally, although in the CBF calculation reported ${ }^{122}$ it was chosen to conform with a two-sublattice Néel ordering, viz., $\mathbf{Q}=\pi / \rho$ for the $1 \mathrm{D}$ chain and $\mathbf{Q}=(\pi / \rho, \pi / \rho)$ for the $2 \mathrm{D}$ square lattice, where $\rho$ is the lattice spacing.

Although the CBF analysis of electron-lattice systems is still very much in its infancy, the results already obtained are very encouraging. The reader is referred to the literature cited for further details.

We turn our attention next to the CCM treatments of Hamiltonian electron-lattice models. ${ }^{109,125-127}$ The calculations performed to date have concentrated on the Hubbard model on the 2D square lattice, at either half-filling ${ }^{109}$ or at half-filling plus one vacancy. ${ }^{125-127}$ They have mostly employed the Néel model state $|N 2\rangle$ as the reference state $|\Phi\rangle$. The half-full electron lattice has then been described by a multiconfigurational correlation operator $S$ as in Eq. (62), and the additional vacancy of momentum $\mathrm{k}$ has been described as a charge-excitation state in terms of an additional multiconfigurational operator $X^{\mathrm{k}}$ as in Eq. (76). The $n$-body partitions of the operators $S=\sum_{n} S_{n}$ and $X^{\mathrm{k}}=\sum_{n} X_{n}^{\mathbf{k}}$ thus involve products of $2 n$ and $(2 n-1)$ single-electron creation operators, respectively, defined with respect to the Néel state as the model vacuum state. For example, the operator $S_{1}$ creates a doubly occupied state and a vacancy; while the operator $S_{2}$ creates (i) two doubly occupied sites and two vacancies, (ii) a spin-flip on a given sublattice and both a doubly occupied site and a vacancy on the other sublattice, and (iii) a spin-exchange between electrons on different sublattices.

As usual in a CCM calculation, the configurations retained in $S$ and $T$ need to be subjected to some systematic approximation hierarchy. Petit and Roger, ${ }^{126}$ who have performed the most ambitious calculations up till now, describe one such localized scheme which is similar to the LSUB $n$ scheme described previously, but which is motivated on quite different grounds. With respect to a model state $|\mathrm{N} 2\rangle$, for which the spins on sites $\{i\}$ on the $A$-sublattice are $\downarrow$ and on sites $\{j\}$ of the $B$-sublattice are $\uparrow$, they consider, for example, the simple approximation,

$$
S \longrightarrow k_{1} \sum_{\langle i, j\rangle}\left(c_{j \downarrow}^{\dagger} c_{i \downarrow}+c_{i \uparrow}^{\dagger} c_{j \uparrow}\right)+k_{2} \sum_{\langle i, j\rangle} c_{j \downarrow}^{\dagger} c_{i \uparrow}^{\dagger} c_{i \downarrow} c_{j \uparrow},
$$

which either creates a double vacancy leaving a neigbouring site empty or exchanges nearest-neighbour spins. They show that for the whole region $U / t \gg 8$, the ground-state energy per electron, $E_{0} / N$, for the half-full $2 \mathrm{D}$ square lattice is only $\approx 2 \%$ above the best quantum Monte Carlo estimates. In particular, in the Heisenberg limit $U / t \rightarrow \infty$, one may readily show that the CCM approximation of Eq. (87) yields $E_{0} / N \rightarrow-\frac{8}{7} J \approx$ $-1.143 J$, where $J \equiv 4 t^{2} / U$ as in the third Section above. By comparison with Eq. (25) this value may be compared with the best available results for the Heisenberg lattice quoted above, $E_{0} / N \approx(-0.669-0.5) J=-1.169 J$. The inclusion of more configurations in Eq. (87) readily yields even better agreement. 
Suppose we now remove an electron on site $i$ from the half-full lattice. Petit and Roger ${ }^{126}$ claim that the most important terms to keep in the excitation operator $X^{\mathbf{k}}$ are now those where the vacancy hops successively between nearest-neighbour sites. For example, keeping only one nearest-neighbour hop, one has

$$
X^{\mathbf{k}} \longrightarrow \sum_{i}\left(c_{i \downarrow}+\kappa \sum_{\rho} c_{i \uparrow}^{\dagger} c_{i \downarrow} c_{i+\rho, \uparrow}\right) \exp \left(i \mathbf{k} \cdot \mathbf{R}_{i}\right)
$$

Petit and Roger keep also terms involving 2 and 3 successive hops between nearest neighbours, and find that a simple extrapolation scheme can be found for the limiting approximation of an infinite number of such successive hops. They present results for the minimum energy and effective mass of a vacancy, which are in good agreement with results obtained for the $t-J$ model through exact diagonalization of small clusters followed by finite-size scaling to the infinite lattice limit.

We note that whereas the above results have all been obtained by the use of the Néel state as the CCM model state, the present author and his colleagues ${ }^{127}$ have also shown how the mean-field state arising from the mean-field theory treatment of the Hubbard model at half filling ${ }^{128}$ might very profitably be used as an alternative model state. Finally, we note that a similar CCM analysis could also be made for the Hubbard models with a negative on-site potential $U$, which can arise from couplings with the lattice phonons. In this case, an obvious model state at half-filling is the so-called charged-density-wave state in which one sublattice is doubly occupied and the other is empty.

\section{Lattice Gauge Theory}

Ristig and his colleagues ${ }^{129}$ have shown how the CBF method may be employed to study the ground and excited states of Abelian and non-Abelian lattice gauge Hamiltonians. They have made a particularly careful and detailed study of the $U(1)$ theory in two spatial dimensions in the uncharged vacuum sector. The ground state is described by a variational trial function of Jastrow type, which includes both single-plaquette terms and two-plaquette correlations. This form is exact in both the strong-coupling and weak-coupling limits, and it incorporates the effects both of virtual lattice photons (or glueballs) and of short-range field correlations.

They evaluate the g.s. energy in terms of a one-plaquette density, a 2-plaquette pair distribution function, and an effective nearest-neighbour plaquette-plaquette interaction. The excited states are represented by trial wave functions of a suitably generalized Bijl-Feynman form. The standard CBF scheme is then implemented to minimize the associated energy expectation values. This procedure thus results in a set of Euler-Lagrange equations to determine the optimized CBF set of correlated basis states, and numerical calculations are performed in a standard hypernetted chain (HNC) approximation.

Using the above procedure, results are firstly obtained for the g.s. energy which compare very favourably with those of alternative calculations, and then for the energies of the lowest branch of elementary excitations and, particularly, for the glueball mass at zero wave vector. They also present results for such other quantities as the singleplaquette density profile, the single-plaquette energy, and the electric and magnetic pair-plaquette correlation functions as functions of the relative plaquette separation. All of the above quantities were calculated for a range of values of the coupling constant $\lambda$ extending from the strong-coupling regime to well into the weak-coupling regime. The results obtained clearly demonstrate the power of the CBF method for applications to Hamiltonian lattice gauge theories in two spatial dimensions. 
The CCM has also been applied to Hamiltonian lattice gauge theories. ${ }^{77,130-132}$ Particular applications have been made to the uncharged $U(1)$ theory in $(1+1),(2+1)$ and $(3+1)$ dimensions, ${ }^{77,130,131}$ the $Z(2)$ model in $(2+1)$ dimensions, ${ }^{131}$ and the $S U(2)$ theory without quarks in $(2+1)$ dimensions. ${ }^{132}$ The applications to the $U(1)$ theory are described in more detail elsewhere in this volume, ${ }^{77}$ and we do not repeat them here. However, the main goal for both the $U(1)$ and $S U(N)$ models is to provide a CCM parametrization of the physical (i.e., gauge-invariant) ground state and excited ("glueball") states of the Hamiltonian of Eq. (35).

We thus need firstly to choose a model state $|\Phi\rangle$. A convenient and obvious first choice is the so-called electric $(\lambda \rightarrow 0)$ vacuum $|0\rangle$, for which $E_{\ell}^{a}|0\rangle=0, \forall \ell, a$. The CCM now parametrizes the exact ground state in terms of this model state as in Eq. (62), with a cluster correlation operator $S$ given by a sum over all possible linearly independent linked clusters of Wilson loops,

$$
S=\sum_{\Gamma} \sum_{p} \mathcal{S}_{\Gamma} A_{\Gamma}(p)
$$

with $\Gamma\left(\equiv\left\{\gamma_{i} ; i=1,2, \cdots, n\right\} ; n=1,2, \cdots\right)$ and $p$ denoting respectively the geometry and position of the oriented loop clusters, and $A_{\Gamma} \equiv A_{\gamma_{1}} \cdots A_{\gamma_{n}}$, with $A_{\gamma} \equiv \operatorname{Tr}[U \cdots U]_{\gamma}$ for each closed loop $\gamma$. It is interesting to note that this exponentiated CCM form of wave function was originally proposed by Greensite ${ }^{133}$ in the context of lattice $S U(N)$ gauge theory. The g.s. energy $E_{0}$ and correlation coefficients $\mathcal{S}_{\Gamma}$ are now determined as in Eqs. (65) and (66) respectively, with $C_{J} \rightarrow A_{\Gamma}^{\dagger}(p)$, and where the inner products incorporate the appropriate group measure. Furthermore, for the Hamiltonian of Eq. (35) and the correlation operator of Eq. (89), the expansion of Eq. (67) terminates at the term of second order in $S$.

The procedure is illustrated for the $U(1)$ model in $(2+1)$ dimensions, where the electric (strong-coupling, $\lambda \rightarrow 0$ ) vacuum is simply a constant. Furthermore, the operator $S$ may be partitioned into $k$-plaquette pieces, $S=\sum_{k=1}^{N_{p}}$, where $N_{p} \rightarrow \infty$ is the total number of elementary square plaquettes on the lattice. We have, for example,

$$
\begin{gathered}
S_{1}=\sum_{n=1}^{\infty} \sum_{p=1}^{N_{p}} \mathcal{S}(n) \cos n B_{p} \\
S_{2}=\sum_{n, n^{\prime}=1}^{\infty} \sum_{p, p^{\prime}=1}^{N_{p}}\left[\mathcal{S}_{p p^{\prime}}^{(1)}\left(n, n^{\prime}\right) \cos n B_{p} \cos n^{\prime} B_{p^{\prime}}+\mathcal{S}_{p p^{\prime}}^{(2)}\left(n, n^{\prime}\right) \sin n B_{p} \sin n^{\prime} B_{p^{\prime}}\right],
\end{gathered}
$$

where the prime on the sum in Eq. (91) excludes the term with $p=p^{\prime}$. For this case we have $S^{\dagger}=S$, and the inner products in the coupled CCM equations (65) and (66) are simply the multiple integrals over all plaquette variables $\left\{B_{p}\right\}$ within the range $-\pi<B_{p} \leq \pi$. We also note that the multi-plaquette correlation operators $S_{k}$ have a close relation to the usual Wilson loops $A_{\Gamma}$ mentioned above. For example, the simple trigonometric relation, $2 \cos B_{1} \cos B_{2}=\cos \left(B_{1}+B_{2}\right)+\cos \left(B_{1}-B_{2}\right)$, and similar addition formulae can easily be used to pass from the representation exemplified by Eqs. (90) and (91) to that of Eq. (89). The former parametrization is not only complete but is also especially useful in view of the orthonormality of the basis.

The multi-plaquette correlations in the exact ground state $\left|\Psi_{0}\right\rangle$ may now be approximated as usual by truncating the operator $S$, using the obvious analogues of the SUB $n$ and LSUB $m$ schemes described previously for the spin-lattice problems, for example. Further sub-truncations can also be made here in terms of the number of modes $\left\{n_{i}\right\}$ kept in the summations of Eqs. (90) and (91) and their higher-order equivalents. 


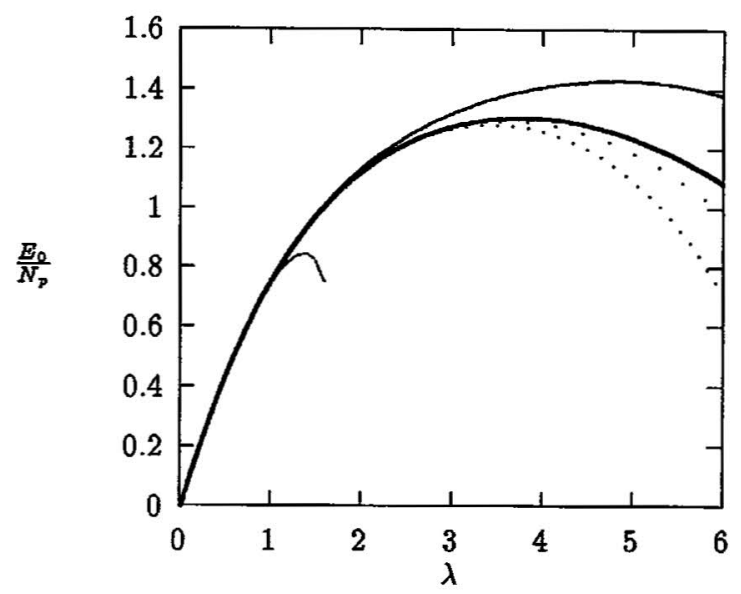

Figure 5. Ground-state energy per plaquette for the $U(1)$ lattice gauge model in $(3+1)$ dimensions for some CCM LSUB2( $k$ ) approximations: $k=2$ (thin line), $k=3$ (medium line), $k=4$ (thick line), $k=5$ (close dots), and $k=6$ (wide dots).

For example, the $\operatorname{LSUB} m(k)$ scheme first sets to zero all terms in $S$ between $n>m$ plaquettes and between $n<m$ plaquettes if those $n$ plaquettes occupy a region which cannot be delimited by at most $m$ contiguous plaquettes on the lattice. Secondly, all terms in the resulting LSUBm correlation operator with $\sum_{i} n_{i}>k$ are also set to zero. The mode numbers $\left\{n_{i}\right\}$ clearly bear a direct relationship with the winding numbers of the equivalent Wilson loops retained in $S$. In this way, it is also clear how the approach may be generalized to the non-Abelian $S U(N)$ lattice gauge theories. Excited states may also be treated in a wholly analogous fashion via the general CCM parametrization of Eqs. (76) and (77).

The full SUB1 calculation for the $U(1)$ model is completely equivalent to the onebody Mathieu problem, and the SUB1 $(k)$ sub-truncations are found to converge extremely rapidly with increasing $k$ even for large values of $\lambda{ }^{130,131}$ The inter-plaquette correlations are then incorporated by including terms in $S=\sum_{n} S_{n}$ with $n>1$. We remark here only that these preliminary investigations of the $U(1)$ lattice gauge theory are very encouraging. It has been shown how specific truncations exactly reproduce strong-coupling $(\lambda \rightarrow 0)$ perturbation theory. They comprise, in effect, a well-defined analytic continuation or resummation of such perturbative series within the context of a natural and consistent hierarchy. They are far superior to the usual (somewhat ad hoc) approaches based on generalized Padé approximants. Ground-state energies and glueball masses are found to be in good agreement with previous accurate results, even for values of the coupling strength as high as $\lambda \sim 10$, which are far into the weakcoupling regime. For details of these and similar calculations of the $Z(2)$ model the reader is referred to the literature. ${ }^{130,131}$ For present purposes we simply show in Fig. 5 the results of some recent CCM calculations for the g.s. energy of the $U(1)$ lattice gauge theory in $(3+1)$ dimensions, using the LSUB2 $(k)$ approximation scheme.

We note that as an alternative to the electric vacuum state $|0\rangle$ as the choice of model state $|\Phi\rangle$, one may also use a mean-field-like model state $|\Phi\rangle \rightarrow\left|\Phi_{\mathrm{mf}}\right\rangle=\mathrm{e}^{T}|0\rangle$, where $T=\frac{1}{2} t \sum_{p=1}^{N_{p}} \cos B_{p}$, and the parameter $t$ is optimized in some suitable fashion, e.g., by minimizing the expectation value $\left\langle\Phi_{\mathrm{mf}}|H| \Phi_{\mathrm{mf}}\right\rangle$. In this way, one may reproduce the exact weak-coupling $(\lambda \rightarrow \infty)$ single-plaquette energy, as well as reproducing the 
lowest-order strong-coupling limit.

Finally, we note that whereas a similar calculation to that described above may be performed for the $S U(2)$ case, Llewellyn Smith and Watson ${ }^{132}$ have given a variant which uses the same basic CCM parametrization of the wave function but which does not use the usual CCM machinery to evaluate the cluster coefficients $\mathcal{S}_{\Gamma}$. In particular, they rearrange the expansion of the truncated wave functions in terms of "shifted" clusters which describe fluctuations about their vacuum expectation values. The resulting nonlinear truncated Schrödinger equations are then solved self-consistently, by a means which avoids the standard CCM projection technique of Eq. (66). In this way they achieve the great advantage of never having to perform any group integrals. Also, all of the Wilson loop vacuum expectation values have the correct weak-coupling form. While the method seems to give good results for the g.s. energy, it is not yet clear whether it is able also to describe accurately the glueball mass, particularly at intermediate to large values of the coupling strength $\lambda$, so that it can be reliably extrapolated to the weak-coupling $(\lambda \rightarrow \infty)$ limit.

\section{CONCLUDING REMARKS}

Although the applications of the CCM and CBF techniques to lattice Hamiltonian systems are still in their infancy, we hope to have convinced the reader that the results obtained to date are encouraging. As was to be expected from their previous successes in describing accurately the properties of a wide range of more traditional forms of condensed matter (such as the liquids helium, the electron gas, nuclear matter, and finite nuclei, atoms and molecules), both methods clearly give very accurate results for such quantities as the ground-state energy. Indeed, we have seen that the CCM, for example, is now capable in the case of spin-lattice Hamiltonians of achieving accuracy comparable to that of the largest Monte Carlo simulations yet performed.

Furthermore, both the CBF and CCM calculations can be done from the outset in the thermodynamic bulk limit, $N \rightarrow \infty$. They do not, therefore, suffer from the finite-size problems inherent in most other methods in common use, especially the exact diagonalizations of small clusters, but also the Monte Carlo methods. Neither are they plagued by the infamous "fermion sign" problem of the latter.

Nevertheless, accuracy for such quantities as the total energy is only part of the story - indeed, only a relatively minor part. We already know that dramatic changes in the wave function often lead to only very minor changes in the estimates for the energy. The resonating valence bond wave function of Liang et al. ${ }^{82}$ for the spin- $\frac{1}{2}$ Heisenberg model on a $2 \mathrm{D}$ square lattice has already been noted in the previous Section in this context. This is a typical example of where, in a variational method, the correlations present in the trial wave function totally prejudice the predictive outcome with regard to the much more subtle and more interesting questions concerning the ordering properties of the ground and excited states.

Many of these subtleties can, however, be addressed by implementing the full CBF machinery discussed above. This will require the calculation of non-diagonal matrix elements of the Hamiltonian and the unit operator in a nonorthogonal complete basis of CBF states, in complete analogy to what has already been achieved for stronglyinteracting continuous systems. In this way the dependence on the starting trial function of the answers to such delicate questions as whether a given model exhibits Fermi liquid or Luttinger liquid behaviour, or as to the details of its critical behaviour near a phase transition, can be overcome. 
Similarly, within the CCM, we have already seen how critical behaviour can be discussed in a systematic and unbiased manner. Nevertheless, even here one will always need in practice to be guided to what are likely to be the most important classes of multiparticle cluster configurations to retain at a given level of approximation for the basis cluster operators of the theory, with respect to a specific model state. Equivalently, there will always remain a use and a need for external guidance on the construction of new hierarchical and systematic CCM truncation schemes.

Thus, we see that while both the CCM and CBF formalisms can provide systematic and unbiased means to discuss the detailed properties of lattice Hamiltonian systems, nevertheless they can (and probably should) be guided from the outset by some external physical knowledge or intuition. We believe that this is likely to remain one of their most important features for future applications. Indeed, by this means they now already provide an ideal forum in which the "conceptual" and "quantitative" schools of quantum many-body theory can begin to talk to each other and thereby hope to interact profitably.

Finally, we note that the CBF and CCM machinery discussed in this paper has been restricted to a description of pure quantum-mechanical states, and hence to zerotemperature properties. Nevertheless, extensions of the formalism to deal with quantum many-body systems at nonzero temperatures have been made for both the CBF method $^{134}$ and the CCM. ${ }^{135}$ We expect to see in the next few years applications of these finite-temperature extensions to deal with accurate descriptions of the phase diagrams and other temperature-dependent properties of lattice Hamiltonian systems.

\section{ACKNOWLEDGMENT}

This work was supported by a research grant awarded by the Science and Engineering Research Council (SERC) of Great Britain, which we gratefully acknowledge.

\section{REFERENCES}

1. J.G. Bednorz and K.A. Müller, Z. Phys. B64:189(1986); Rev. Mod. Phys. 60:585(1988).

2. P.W. Anderson, Science 235:1196(1987); in: "Frontiers and Borderlines in ManyBody Physics," J.R. Schrieffer and R.A. Broglia, ed., North-Holland, Amster$\operatorname{dam}(1987)$.

3. J. Hubbard, Proc. R. Soc. London Ser. A 276:238(1963); ibid. 281:401(1964).

4. H.G. Kümmel, in: "Recent Progress in Many-Body Theories," Vol. 2, Y. Avishai, ed., Plenum, New York (1990), p. 353.

5. L. Yu, in: "Recent Progress in Many-Body Theories," Vol. 3, T.L. Ainsworth, C.E. Campbell, B.E. Clements, and E. Krotscheck, ed., Plenum, New York (1992), p. 157.

6. F. Coester, Nucl. Phys. 7:421(1958); F. Coester and H. Kümmel, ibid. 17:477(1960).

7. J. Čižek, J. Chem. Phys. 45:4256(1966); Advan. Chem. Phys. 14:35(1969).

8. H. Kümmel, K.H. Lührmann, and J.G. Zabolitzky, Phys. Rep. 36C:1(1978).

9. R.F. Bishop and K.H. Lührmann, Phys. Rev. B17:3757(1978); ibid. B26:5523(1982).

10. H.G. Kümmel, in: "Nucleon-Nucleon Interaction and Nuclear Many-Body Problems," S.S. Wu and T.T.S. Kuo, ed., World Scientific, Singapore (1984), p. 46.

11. R.F. Bishop and H.G. Kümmel, Phys. Today 40(3):52(1987).

12. J.S. Arponen, R.F. Bishop, and E. Pajanne, Phys. Rev. A36:2519(1987); ibid. 
A36:2539(1987).

13. R.F. Bishop, Theor. Chim. Acta 80:95(1991).

14. R.F. Bishop, in: "Dirkfest '92-A Symposium in Honor of J. Dirk Walecka's Sixtieth Birthday," W.W. Buck, K.M. Maung, and B.D. Serot, ed., World Scientific, Singapore (1992), p. 21.

15. R.F. Bishop, in: "Many-Body Physics," C. Fiolhais, M. Fiolhais, C. Sousa, and J.N. Urbano, ed., World Scientific, Singapore (1994), p. 3.

16. E. Feenberg, "Theory of Quantum Fluids," Academic Press, New York (1969).

17. J.W. Clark and E. Feenberg, Phys. Rev. 113:388(1959); H.W. Jackson and E. Feenberg, Ann. Phys. (NY) 15:266(1961); E. Feenberg and C.W. Woo, Phys. Rev. 137:A391(1965); J.W. Clark and P. Westhaus, Phys. Rev. 141:833(1966); ibid. 149:990(1966).

18. J.W. Clark, L.R. Mead, E. Krotscheck, K.E. Kürten, and M.L. Ristig, Nucl. Phys. A328:45(1979); E. Manousakis and V.R. Pandharipande, Phys. Rev. B30:5062 (1984).

19. E. Krotscheck and J.W. Clark, Nucl. Phys. A328:73(1979).

20. J.W. Clark, Prog. Part. Nucl. Phys. 2:89(1979).

21. J.W. Clark, in: "The Many-Body Problem, Jastrow Correlations Versus Brueckner Theory," R. Guardiola and J. Ros, ed., (Lect. Notes in Physics, Vol. 138), Springer, Berlin (1981), p. 184.

22. E. Krotscheck, J.W. Clark, and A.D. Jackson, Phys. Rev. B28:5088(1983).

23. J.W. Clark, E. Krotscheck, and B. Schwesinger, Phys. Lett. 143B:287(1984).

24. W. Heisenberg, Z. Phys. 49:619(1928).

25. P.A.M. Dirac, Proc. R. Soc. London Ser. A 123:714(1929).

26. J.H. Van Vleck, "Theory of Electric and Magnetic Susceptibilities," Clarendon Press, Oxford (1932).

27. E. Manousakis, Rev. Mod. Phys. 63:1(1991).

28. T. Barnes, Int. J. Mod. Phys. C2:659(1991).

29. F.D.M. Haldane, Phys. Lett. 93A:464(1983); Phys. Rev. Lett. 50:1153(1983).

30. I. Affleck, J. Phys.: Condens. Matter 1:3047(1989).

31. D.S. Greywall and P.A. Busch, Phys. Rev. Lett. 65:2788(1990); D.S. Greywall, Phys. Rev. B41:1842(1990).

32. H.A. Bethe, Z. Phys. 71:205(1931).

33. L. Hulthén, Ark. Mat. Astron. Fys. A26, No. 11 (1938).

34. R. Orbach, Phys. Rev. 112:309(1958); C.N. Yang and C.P. Yang, ibid. 150:321(1966); ibid. 150:327(1966).

35. M. Gaudin, "La Fonction d'Onde de Bethe," Masson, Paris (1983).

36. J. des Cloiseaux and J.J. Pearson, Phys. Rev. 128:2131(1962); J.D. Johnson, S.

Krinsky, and B.M. McCoy, Phys. Rev. A8:2526(1973); L.D. Faddeev and L.A. Takhtajan, Phys. Lett. 85A:375(1981).

37. R.J. Baxter, J. Stat. Phys. 9:145(1973).

38. N.M. Bogoliubov, A.G. Izergin, and V.E. Korepin, Nucl. Phys. B275:687(1986).

39. E.K. Sklyanin, L.A. Takhtajan, and L.D. Faddeev, Theor. Math. Phys. 40:688(1980);

V.E. Korepin, N.M. Bogoliubov, and A.G. Izergin, "Quantum Inverse Scattering Method and Correlation Functions," Cambridge Univ. Press (1993).

40. A. Luther and I. Peschel, Phys. Rev. B12:3908(1975).

41. F.J. Dyson, E.H. Lieb, and B.J. Simon, J. Stat. Phys. 18:335(1978).

42. E. Jordao Neves and J. Fernando Perez, Phys. Lett. 114A:331(1986);

I. Affleck, T. Kennedy, E.H. Lieb, and H. Tasaki, Phys. Rev. Lett. 59:799(1987);

Commun. Math. Phys. 115:447(1988); T. Kennedy, E.H. Lieb, and B.S. 
Shastry, J. Stat. Phys. 53:1019(1988).

43. B. Bernu, P. Lecheminant, C. Lhuillier, and L. Pierre, Phys. Scripta T49:192(1993);

Phys. Rev. B50:10048(1994).

44. J. Carlson, Phys. Rev. B40:846(1989); N. Trivedi and D.M. Ceperley, ibid. B41:4552 (1990); K.J. Runge, ibid. B45:12292(1992).

45. P.W. Anderson, Mater. Res. Bull. 8:153(1973); P. Fazekas and P.W. Anderson, Phil. Mag. 30:423(1974).

46. K. Kubo and T. Kishi, Phys. Rev. Lett. 61:2585(1988).

47. C.K. Majumdar and D.K. Ghosh, J. Math. Phys. 10:1388(1969); ibid. 10:1399(1969).

48. F.D.M. Haldane, Phys. Rev. B25:4925(1982).

49. L.A. Takhtajan, Phys. Lett. 87A:479(1982); H.M. Babujian, ibid. 90A:479(1982).

50. C.K. Lai, J. Math. Phys. 15:1675(1974); B. Sutherland, Phys. Rev. B12:3795(1975).

51. J.B. Parkinson, J. Phys. C20:L1029(1987); ibid. C21:3793(1988); M.N. Barber and

M.T. Bachelor, Phys. Rev. B40:4621(1989); A. Klümper, J. Phys. A23:809(1990).

52. A.V. Chubukov, J. Phys.: Condens. Matter 2:1593(1990); Phys. Rev. B47:872(1993).

53. G. Fáth and J. Sólyom, Phys. Rev. B44:11836(1991); ibid. B47:872(1993).

54. T. Tonegawa and I. Harada, J. Phys. Soc. Japan 56:2153(1987).

55. A.B. Harris and R.V. Lange, Phys. Rev. 157:279(1967); W.F. Brinkman and T.M. Rice, Phys. Rev. B2:1324(1970); J.E. Hirsch, Phys. Rev. Lett. 54:1317(1985).

56. V.J. Emery, Phys. Rev. Lett. 58:2794(1987); J.E. Hirsch, ibid. 59:228(1987); P.B.

Littlewood, C.M. Varma, and E. Abrahams, ibid. 60:379(1987).

57. F.C. Zhang and T.M. Rice, Phys. Rev. B37:3759(1988); ibid. B41:7243(1990).

58. V.J. Emery and G. Reiter, Phys. Rev. B38:11938(1988); ibid. B41:7247(1990).

59. E. Dagotto, Rev. Mod. Phys. 66:763(1994).

60. P.W. Anderson, in: "Valence Fluctuations in Solids," L.M. Falicov, W. Hanke, and M.M. Maple, ed., North-Holland, Amsterdam (1981), p. 451; C.M. Varma, Comments Solid State Phys. 11:221(1985).

61. L. Yu, "Solitons and Polarons in Conducting Polymers," World Scientific, Singapore (1988).

62. D. Jerome and H.J. Schultz, Adv. Phys. 31:299(1982).

63. E.H. Lieb and F.Y. Wu, Phys. Rev. Lett. 20:1445(1968).

64. F.D.M. Haldane, Phys. Rev. Lett. 45:1358(1980); J. Phys. C14:2585(1981); Phys. Lett. 81A:153(1981).

65. J.M. Luttinger, J. Math. Phys. 4:1154(1963).

66. K.G. Wilson, Phys. Rev. D10:2445(1974).

67. J. Kogut and L. Susskind, Phys. Rev. D11:395(1975).

68. S. Elitzur, Phys. Rev. D12:3978(1975).

69. A.M. Polyakov, Phys. Lett. B72:477(1978).

70. E. Fradkin and L. Susskind, Phys. Rev. D17:2637(1978).

71. S.D. Drell, H.R. Quinn, B. Svetitsky, and M. Weinstein, Phys. Rev. D19:619(1979).

72. R. Balian, J.M. Drouffe, and C. Itzykson, Phys. Rev. D10:3376(1974); ibid.

D11:2098(1975); ibid. D11:2104(1975).

73. D. Horn, M. Weinstein, and S. Yankielowicz, Phys. Rev. D19:3715(1979).

74. F.J. Wegner, J. Math. Phys. 12:2259(1971).

75. S.F. Edwards and P.W. Anderson, J. Phys. F5:965(1975).

76. A.A. Migdal, Sov. Phys. - JETP 42:413(1976); ibid. 42:743(1976).

77. R.F. Bishop, N.J. Davidson, and Y. Xian, article in this volume.

78. J.B. Kogut, Phys. Rep. 67:67(1980).

79. H.G. Dosch and V.F. Müller, Fortschr. Phys. 27:547(1979).

80. I. Montvay and G. Münster, "Quantum Fields on a Lattice," Cambridge Univ. 
Press (1994).

81. C.J. Hamer, J. Oitmaa, and W.H. Zheng, Phys. Rev. D43:1978(1991).

82. S.B. Liang, B. Doucot, and P.W. Anderson, Phys. Rev. Lett. 61:365(1988).

83. D. Horn and M. Weinstein, Phys. Rev. D30:1256(1984).

84. K.G. Wilson, Nucl. Phys. B (Proc. Suppl.) 17:82(1990).

85. R.J. Bartlett, J. Phys. Chem. 93:1697(1989); Theor. Chim. Acta 80:71(1991).

86. R. Jastrow, Phys. Rev. 98:1479(1955).

87. F. Iwamoto and M. Yamada, Prog. Theor. Phys. 17:543(1957).

88. J.W. Clark and P. Westhaus, J. Math. Phys. 9:131(1968); P. Westhaus and J.W. Clark, ibid. 9:149(1968).

89. M. Gaudin, J. Gillespie, and G. Ripka, Nucl. Phys. A176:237(1971); S. Fantoni and S. Rosati, Nuovo Cim. 20A:179(1974); G. Ripka, Phys. Rep. 56:1(1979); Nucl. Phys. A314:115(1979).

90. S. Fantoni and S. Rosati, Lett. Nuovo Cim. 10:545(1974); Nuovo Cim. 25A:593 (1975);E. Krotscheck and M.L. Ristig, Phys. Lett. 48A:17(1974); Nucl. Phys. A242:389(1975).

91. J.W. Clark, in: "Progress in Particle and Nuclear Physics," Vol. 2, D.H. Wilkinson, Pergamon, Oxford (1979), p. 89.

92. S. Rosati and M. Viviani, in: "First International Course on Condensed Matter," D. Prosperi, S. Rosati, and G. Violini, ed., (ACIF Series, Vol. 8), World Scientific, Singapore (1988), p. 231.

93. (a) R.F. Bishop, in: "Recent Progress in Many-Body Theories," Vol. 1, A.J. Kallio, E. Pajanne, and R.F. Bishop, ed., Plenum, New York (1988), p. 385;

(b) A. Fabrocini and S. Rosati, in: "First International Course on Condensed Matter," D. Prosperi, S. Rosati, and G. Violini, ed., (ACIF Series, Vol. 8), World Scientific, Singapore (1988), p. 87.

94. J.W. Clark and M.L. Ristig, Phys. Rev. 7:1792(1973); M.L. Ristig and J.W. Clark, Nucl. Phys. A199:351(1973).

95. L.R. Mead and J.W. Clark, Phys. Lett. 90B:331(1980).

96. J. Arponen, Ann. Phys. (NY) 151:311(1983).

97. K. Emrich, Nucl. Phys. A351:379(1981); ibid. A351:397(1981).

98. A.D. Jackson, A. Lande, and R.A. Smith, Phys. Rev. Lett. 54:1469(1985); E. Krotscheck, R.A. Smith, and A.D. Jackson, Phys. Rev. A33:3535(1986).

99. P.W. Kastelijn, Physica 18:104(1952).

100. W. Marshall, Proc. R. Soc. London Ser. A 232:48(1955).

101. H. Taketa and T. Nakamura, J. Phys. Soc. Japan 11:919(1956); R. Bartkowski, Phys. Rev. B5:4536(1972); M. Suzuki and S. Miyashita, Can. J. Phys. 56:902 (1978); R.B. Pearson, Phys. Rev. A18:2655(1978).

102. P. Horsch and W. von der Linden, Z. Phys. B72:181(1988); D.A. Huse and V. Elser, Phys. Rev. Lett. 60:2531(1988); Z. Liu and E. Manousakis, Phys. Rev. B40:11437(1989).

103. S. Sachdev, Phys. Rev. B39:12232(1989); T. Pang, ibid. B43:3362(1991).

104. E. Manousakis, Phys. Rev. B40:4904(1989).

105. P.W. Anderson, Phys. Rev. 86:694(1952); R. Kubo, ibid. 87:568(1952); T. Oguchi, ibid. 117:117(1960).

106. J.E. Hirsch and S. Tang, Phys. Rev. B40:4769(1989); S. Tang, M.E. Lazzouni, and J.E. Hirsch, ibid. B40:5000(1989).

107. M. Takahashi, Phys. Rev. B40:2494(1989).

108. M. Roger and J.H. Hetherington, Phys. Rev. B41:200(1990).

109. M. Roger and J.H. Hetherington, Europhys. Lett. 11:255(1990). 
110. R.F. Bishop, J.B. Parkinson, and Y. Xian, (a) Phys. Rev. B43:13782(1991);

(b) Theor. Chim. Acta 80:181(1991); (c) Phys. Rev. B44:9425(1991); (d) in:

"Recent Progress in Many Body Theories," Vol. 3, T.L. Ainsworth, C.E. Campbell, B.E. Clements, and E. Krotscheck, ed., Plenum, New York (1992), p. 117;

(e) J. Phys.: Condens. Matter 4:5783(1992).

111. R.F. Bishop, J.B. Parkinson, and Y. Xian, Phys. Rev. B46:880(1992).

112. R.F. Bishop, J.B. Parkinson, and Y. Xian, J. Phys.: Condens. Matter 5:9169(1993).

113. F.E. Harris, Phys. Rev. B47:7903(1993).

114. (a) D.J.J. Farnell and J.B. Parkinson, J. Phys.: Condens. Matter 6:5521(1994); (b) Y. Xian, ibid. 6:5965(1994).

115. F. Cornu, Th. Jolicoeur, and J.C. Le Guillou, Phys. Rev. B49:9548(1994).

116. R.F. Bishop, R.G. Hale, and Y. Xian, Phys. Rev. Lett. 73:3157(1994); UMIST preprint (1994).

117. T. Barnes, D. Kotchan, and E.S. Swanson, Phys. Rev. B39:4357(1989).

118. R.R.P. Singh, Phys. Rev. B39:9760(1989); R.R.P. Singh and D.A. Huse, ibid. B40:7247(1989); W. Zheng, J. Oitmaa, and C.J. Hamer, ibid. B43:8321(1991).

119. B.I. Shraiman and E.D. Siggia, Phys. Rev. Lett. 61:467(1988); ibid. 62:1564(1989).

120. H. Yokoyama and H. Shiba, J. Phys. Soc. Japan 56:3570(1987); C. Gros, R. Joynt, and T.M. Rice, Phys. Rev. B36:381(1987); S. Fantoni, X.Q. Wang, E. Tosatti, and L. Yu, Physica C153-155:1255(1988).

121. X.Q. Wang, S. Fantoni, E. Tosatti, L. Yu, and M. Viviani, Phys. Rev. B41:11479 (1990).

122. X.Q. Wang, S. Fantoni, E. Tosatti, and L. Yu, in: "Condensed Matter Theories," Vol. 5, V.C. Aguilera-Navarro, ed., Plenum, New York (1990), p. 203; X.Q.

Wang, S. Fantoni, E. Tosatti, L. Yu, and X.Q.G. Wang, in: "Recent Progress in Many-Body Theories," Vol. 2, Y. Avishai, ed., Plenum New York (1990), p. 297.

123. T.A. Kaplan, P. Horsch, and P. Fulde, Phys. Rev. Lett. 49:889(1982); P. Fazekas and K. Penc, Int. J. Mod. Phys. B1:1021(1988).

124. C.H. Yang and J.W. Clark, Nucl. Phys. A174:49(1971); E. Krotscheck and J.W. Clark, ibid. A333:77(1980); T.C. Paulick and C.E. Campbell, Phys. Rev. B16:2000(1977); S. Fantoni, Nucl. Phys. A363:381(1981).

125. C.F. Lo, E. Manousakis, and Y.L. Wang, Phys. Lett. 156A:42(1991).

126. F. Petit and M. Roger, Phys. Rev. B49:3453(1994).

127. R.F. Bishop, Y. Xian, and C. Zeng, Int. J. Quantum Chem. - in press.

128. J.R. Schrieffer, X.G. Wen, and S.C. Zhang, Phys. Rev. B39:11663(1989).

129. A. Dabringhaus and M.L. Ristig, in: "Condensed Matter Theories," Vol. 6, S.

Fantoni and S. Rosati, ed., Plenum, New York (1991), p. 291; A. Dabring-

haus, M.L. Ristig, and J.W. Clark, Phys. Rev. D43:1978(1991); M.L. Ristig and A. Dabringhaus, in: "Recent Progress in Many-Body Theories," Vol. 3, T.L. Ainsworth, C.E. Campbell, B.E. Clements, and E. Krotscheck, ed., Plenum, New York (1992), p. 107.

130. R.F. Bishop, A.S. Kendall, L.Y. Wong, and Y. Xian, Phys. Rev. D48:887(1993); in: "Condensed Matter Theories," Vol. 8, L. Blum and F.B. Malik, ed., Plenum, New York (1993), p. 269.

131. R.F. Bishop and Y. Xian, Acta Phys. Pol. 24:541(1993); Nucl. Phys. B (Proc. Suppl.) 34:808(1994).

132. C.H. Llewellyn Smith and N.J. Watson, Phys. Lett. B302:463(1993).

133. J.P. Greensite, Nucl. Phys. B166:113(1980).

134. C.E. Campbell, K.E. Kürten, M.L. Ristig, and G. Senger, Phys. Rev. B30:3728 
(1984); G. Senger, M.L. Ristig, K.E. Kürten, and C.E. Campbell, Phys. Rev. B33:7562(1986); G. Senger and M.L. Ristig, in: "Condensed Matter Theories," Vol. 5, V.C. Aguilera-Navarro, ed., Plenum, New York (1990), p. 133; C.E. Campbell, in: "Condensed Matter Theories," Vol. 8, L. Blum and F.B. Malik, ed., Plenum, New York (1992), p. 149.

135. M. Altenbokum, K. Emrich, H. Kümmel, and J.G. Zabolitzky, in: "Condensed Matter Theories," Vol. 2, P. Vashishta, R.K. Kalia, and R.F. Bishop, ed., Plenum, New York (1987), p. 389; G. Sanyal, S.H. Mandal, and D. Mukherjee, Chem. Phys. Lett. 192:55(1992); G. Sanyal, S.H. Mandal, S. Guha, and D. Mukherjee, Phys. Rev. E48:3373(1993). 